Brösel, Gerrit ; Dechant, Hubert:

Ein Ansatz zur Bewertung von Telekommunikationsunternehmungen und von deren abgrenzbaren Unternehmungsteilen

Zuerst erschienen in:

E-Business, M-Business und T-Business : digitale Erlebniswelten aus Sicht von Consulting-Unternehmen. - Wiesbaden : Gabler, (2003), S. $133-166$ 
Frank Keuper (Hrsg.)

\section{E-Business, M-Business \\ und T-Business}

Digitale Erlebniswelten aus Sicht von Consulting-Unternehmen

Sonderdruck

(c) Betriebswirtschaftlicher Verlag Dr. Th. Gabler/GWV Fachverlage $\mathrm{GmbH}$, Wiesbaden 2003 


\title{
Ein Ansatz zur Bewertung von Telekommunikationsunternehmungen und von deren abgrenzbaren Unternehmungsteilen
}

\author{
GERRT BRÖSEL und HuBERT DECHANT
}

PwC DEUTSCHE REVISION SCHWERIN Und TECHNISCHE UNIVERSITÄT ILMENAU

$1 \quad$ Die Problemstellung

2 Die Grundlagen der Bewertung

2.1 Die Bewertungsanlässe

2.2 Die Konzeptionen der Bewertung 138

2.3 Die Funktionen der Bewertung und ihre Wertarten 140

2.4 Die Grundsätze der Bewertung von Telekommunikationsunternehmungen und von deren Unternehmungsteilen

3 Die Abgrenzung der Zukunftserfolge 143

3.1 Die grundlegenden Prinzipien zur Abgrenzung der Zukunftserfoige $\quad 143$

3.2 Ein Ansatz zur Prognose der Zukunftserfolge $\quad 146$

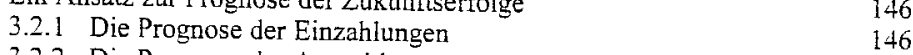

$\begin{array}{ll}\text { 3.2.2 Die Prognose der Auszahlungen } & 151\end{array}$

4 Die Transformation der Zukunftserfolge 155

4.1 Das Zukunftserfolgswertverfahren 155

$\begin{array}{ll}4.2 \text { Die Berücksichtigung der Unsicherheit } & 155 \\ & \end{array}$

5 Eine Zusammenfassung $\quad 159$

$\begin{array}{ll}\text { Symbolverzeichnis } & 159\end{array}$

$\begin{array}{ll}\text { Quellenverzeichnis } & 162\end{array}$ 
Die Euphorie, die vor, während und teilweise auch nach der Versteigerung der „Universal Mobile Telecommunications System"-Lizenzen (UMTS-Lizenzen)' im Jahre 2000 auf den Seiten der Telekommunikationsuntemehmungen herrschte, scheint verflogen. Emüchterung macht sich breit, und mindestens zwei der sechs UMTS-Lizenznehmer haben bereits ihre Hoffnungen begraben. ${ }^{2}$ "Wir haben die Chancen von UMTS eindeutig überschätzt" ${ }^{* 3}$, ist vom Vorstandsvorsitzenden der MOBILCOMAG. Herm DR. THORSTEN GRENZ, zu vernehmen. Hinsichtlich der bei der UMTS-Auktion erzielten astronomischen Preise und der derzeitigen wirtschaftlichen Probleme einiger Lizenznehmer ist es fraglich, ob die präsumtiven Lizenznehmer ihre maximal zahlbaren Preise vor der Gebotsabgabe mithilfe theoretisch fundierter Methoden ermittelt haben oder diese Grenzpreise überhaupt kannten. Gibt eine Telekommunikationsunternehmung ihre UMTS-Pläne auf, fällt die Lizenz an den Regulierer zurück, wenn diese Unternehmung nicht bis zum 31. Dezember 2003 über ein UMTS-Netz verfügt, welches mindestens $25 \%$ der Bevölkerung erreicht. Ein Verkauf der einzelnen Lizenz ist vertraglich ausgeschlossen. Nunmehr hoff jedoch die WOBILCOMAG darauf, ihre Tochterunternehmung MOBILCOM MULTIMEDLA GMBH, die sowohl über das bisher fertiggestellte UMTSNetz als auch über die UMTS-Lizenz verfügt, an einen Interessenten verkaufen zu können. ${ }^{+}$

Im Mittelpunkt der nachfolgenden Ausfïhrungen steht deshalb die Bewertung von Telekommunikationsunternehmungen und von ihren abgrenzbaren Unternehmungsteilen, also auch von einer UMTS-Lizenz, vor deren Erwerb: Welchen Preis kann der rational handelnde präsumtive Erwerber ${ }^{j}$ einer Telekommunikationsunternehmung oder einer UMTS-Lizenz maximal für diese zahlen, ohne sich durch den Erwerb schlechter zu stellen als bei Unterlassung dieser Handlung? Unter dem Begriff des "Wertes" soll eine Subjekt-Objekt-ObjektBeziehung verstanden werden. ${ }^{5}$ Der Wert drückt aus, welchen Nutzen sich der präsumtive Erwerber - das Bewertungssubjekt - aus dem Bewertungsobjekt im Hinblick auf die zur Verfügung stehenden Vergieichsobjekte verspricht. ${ }^{7}$ Der zu ermittelnde Wert hat den Charakter einer Entscheidungsgrenze und ist abhängig vom Zielsystem sowie vom Entscheidungsfeld des Bewertungssubjekts. Der Erwerb der Telekommunikationsunternehmung oder von dessen Unternehmungsteilen ist ökonomisch nicht nachteilig, wenn dessen Wert mindestens dem zu zahlenden Preis entspricht. ${ }^{8}$

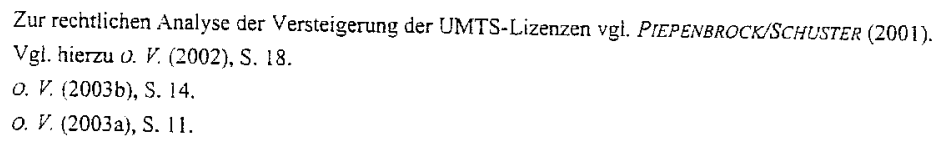

5 Rationales Handein erfordert jedoch unabhängig von der gewählten Marktinstitution (also auch bei einer Auktion) eine Bewertung des zu erwerbenden oder zu veräußernden (also auch des zu er-oder versteigemden) Objekts durch die präsumtiven Erwerber und die präsumtiven Veräußerer. Vgi. zur Auktion REICHWALDiHERMANN/ BIEBERBACH (2000).

6 Vgl. hierzu MATSCHKE (1972), S. 147 und SIEBEN (1988), S. 87.

3 Der Wert eines Gutes ergibt sich hinsichtlich des Ziel- und Präferenzsystems des Bewertungssubjekts gemäß der von HERMANN HEINR/CH GOSSEN Sowie CARL MENGER (und der so genannten Wiener Schule) begrïndeten subjektiven Wertlehre aus seinem Grenznutzen und ist somit individuell (das heißt subjektiv); vgl. HERING (2000), S. 435. Siehe zu den Ursprüngen der subjektiven Werlehre GOSSEN (1854) und MEVGER (1871).

8 Vgl. HERING (1999), S. 1. Zur Abgrenzung von Wert und Preis siehe ENGELS (1962), S. 37 ff., MÜNSTERMANN (1966), S. 151, OLBRICH (2000), S. 459. 
Die Bewertung wird im entscheidungsorientierten Sinne in folgende Schritte unterteilt:

1. Abgrenzung und Quantifizierung der relevanten Zukunftserfolge

2. Transformation der ermittelten Zukunftserfolge in einen Entscheidungswert

3. Abwägung von (subjektivem) Wert und (objektivem) Preis

Die Abgrenzung und Quantifizierung der retevanten Zukunftserfolge wird im Rahmen der Unternehmungsbewertungstheorie bislang vernachlässigt. Sie obliegt den jeweiligen Fachleuten der Branche. Die Charakteristika des Telekommunikationsmarktes verschärfen die ohnehin vorliegenden Ermittlungsprobleme: Schnelllebigkeit, hohe Wettbewerbsintensität und rasanter technologischer Wandel zeichnen den Telekommunikationsmarkt aus. Vergangenheitsorientierte mathematisch-statistische Prognoseverfahren erweisen sich als unbrauchbar. Als Hauptaufgabe der Bewertung wird die Transformation der aus fundierten Schätzungen ermittelten qualitativen und quantitativen Informationen über künftige Erfolge in einen Wert, der die mit der Bewertung verfolgte Funktion erfüllt, angesehen. ${ }^{9}$ Dieser Wert soll dem Entscheidungssubjekt zur Entscheidungsunterstützung dienen. Die nicht formalisierbare Abwägung zwischen Preis und Entscheidungswert, in welche die individueilen Risikoneigungen des Entscheidungssubjekts einfließen, schließt sich an und erfordert transparente Informationsgrundlagen. ${ }^{10}$

Ziel dieser Ausführungen ist die Unterstützung der ersten zwei Bewertungsschritte. Hierzu werden im zweiten Kapitel die Grundlagen der Bewertung dargestellt. Nach einer einleitenden Systematisierung von Bewertungsanlässen wird die Enrwicklung der Unternehmungsbewertungstheorie von der objektiven über die subjektive zur funktionalen Konzeption beschrieben. Wie jede Rechnung muss auch die Bewertungsrechnung zweckorientiert erfolgen. Die sich anschließenden Ausführungen geben deshalb einen Überblick über die Hauptfunktionen der herrschenden funktionalen Bewertungslehre und ihre Wertarten. Schließlich werden die Grundsätze der Bewertung von Telekommunikationsunternehmungen und von deren abgrenzbaren Unternehmungsteilen vorgestellt. Das dritte Kapitel befasst sich mit der bisher in der Bewertungsliteratur zumeist vernachlässigten Abgrenzung und Quantifizierung der relevanten Zukunftserfolge. Dabei werden einerseits die bei der Ermittlung der zukünftigen Erfolge zu beachtenden grundlegenden Prinzipien dargestellt und andererseits ein heuristischer Ansatz präsentiert, welcher die Zukunftserfolgsermittlung unterstützt. Bevor die Ergebnisse des Beitrags zusammengefasst werden, wird im vierten Kapitel ein theoretisch fundiertes Modell zur Transformation der Zukunftserfolge in die gesuchte Größe präsentiert, wobei ein besonderes Augenmerk auf die Berücksichtigung der Unsicherheit gelegt wird.

9 Auf Grund der in der Realität herrschenden Unsicherheit über zukünftige entscheidungsrelevante Sachverhalte ist es ex ante nicht möglich, einen eindeutigen Wert zu ermittein. Wird hier von der Ermirtlung eines Wertes gesprochen. welcher der Entscheidungsunterstützung dienen soll, ist unter dem Begriff Entscheidungswert vielmehr eine Bandbreite möglicher Werte zu verstehen; vgl. HERING (1999), S. 2 ff.

10 Siehe auch HERING (1999), S. 26. 


\title{
Die Grundlagen der Bewertung
}

\section{1}

\author{
Die Bewertungsanlässe
}

Die Antässe für Unternehmungsbewertungen und Bewertungen von Unternehmungsteilen sind vielgestaltig. Eine Systematisierung dieser Anlässe, die letztlich die modelltheoretische Analyse unterstützt und adäquate Bewertungsmodelle ableiten lässt, ist auf LATSCHKE zurückzưühren. Anlässe, die im Zusammenhang mit einer Änderung der Rechtsverhältnisse" stehen, lassen sich klassifizieren in Konfliktsituationen vom Typ des Erwerbs/der Veräußerung und vom Typ der Fusion/Spaltung, eindimensionale und mehrdimensionale Konfliktsituationen, jungierte und disjungierte Konfliktsituationen sowie dominierte und nicht dominierte Konfliktsituationen. ${ }^{12}$

Den Konfliktsituationen vom Typ des Erwerbs/der Veräußenning ${ }^{13}$ werden jene Bewertungsanlässe subsumiert, bei denen sich die Rechtsverhältnisse an der zu bewertenden Unternehmung oder an den zu bewertenden Unternehmungsteilen dergestalt ändern sollen, dass entweder eine der Konfliktparteien (Verkäufer und zugleich Veräußerer) ihr Eigentum am Bewertungsobjekt zugunsten einer anderen Konfliktpartei (Käufer und zugleich Erwerber) aufgibt und dafur eine Gegenleistung (Kaufpreis) von dieser erhält oder die eine Partei (Lizenzgeber und zugleich Veräußerer) einer anderen Partei (Lizenzgeber und zugleich Erwerber) das Bewertungssubjekt überträgt und dafür von dieser als Entschädigung eine Geidzahlung (Lizenzentgelt) erhält. ${ }^{4}$ In der Konfliktsituation vom Typ der Fusion/Spaltung kommt es hingegen nicht zu einem Wechsel der Eigentümer. Von einer Konfliktsituation vom Typ der Fusion $^{15}$ wird gesprochen, wenn mehrere Untemehmungen vereinigt werden sollen und die Eigentümer dieser zu bewertenden Untemehmungen Eigentum an der aus der Vereinigung entstehenden wirtschaftlichen Einheit erhalten werden. ${ }^{16}$ Spiegelbildlich zur Fusion lässt sich die Konfliktsituation vom Typ der Spaltung beschreiben. ${ }^{17}$

II Vgl. SIEBEN (1993), Sp. 4320 f. zu Unternehmungsbewertungsanlässen ohne Änderung der Eigennumsverhältnisse, worunter z. B. Untemehmungsbewerungen in Rahmen der Ermittlung von steuerlichen Bemessungsgrundlagen zählen. Wie BORNER dabei von entscheidungsunabhängigen Aniässen zu sprechen, ist nicht gerechtfertigt, weil beispielsweise auch Bewertungen auf Grund von Kreditwürdigkeitsprütungen durchaus Entscheidungen nach sich ziehen; vgl. BÖRNER (1980), S. $112 \mathrm{ff}$.

12 Vgl. MATSCHKE (1975), S. 33 ff; zur Unterscheidung in dominierte und nicht dominierte Konfiktsituationen vgl. MLATSCHKE (1979), S. 30 ff. Der Typ der Fusion wurde um den Typ der Spaitung ergänzt durch MANDLR $t B E L$ (1997), S. 14 f. Siehe zur graphischen Übersicht möglicher Ausprägungen von Konfliktsituationen OLBRICH (1999), S. 13.

13 Un in die Bewertungsanlässe auch die Ersteigerung von Lizenzen (z. B. UMTS-Lizenz) zu integrieren, wurden die Konfliktsituationen vom Typ des Kaufs/Verkaufs und der LizenzmahmeiLizenzvergabe - in enger Anlehnung an MATSCAKE - zur Konfliktsituation vom Typ des Erwerbs und der Veräußerung zusammengefasst. Vgl. zu dieser Erweiterung auch BRÖSEL (2002), S. 54.

14 Vgl. MATSCHKE (1975), S. 31.

is Zu den Verfahren der Unternehnungsbewertung im Rahmen der Fusion vgl. MATSCHKE (1975), S. 327 ff., MATSCHKE (1984), REICHERTER (2000).

16 Vgl. MATSCHKE (1975), S. 31.

17 Vgl. MANDL/RABEL (1997), S. $14 \mathrm{f}$. 
Um in Verhandlungssituationen eine Einigung zwischen den konfligierenden Parteien herbeizuführen, ist eine Verständigung über bestimmte Bedingungen erforderiich. Diese werden ais konfliktlösungsrelevante Sachverhaite bezeichnet. Hinsichtlich der Anzahl dieser Einigungsbedingungen wird in eindimensionale und mehrdimensionale Konfliktsituationen unterschieden. ${ }^{18}$ Von eindimensionalen Konfliktsituationen wird gesprochen. wenn für die Einigung der konfligierenden Parteien lediglich ein konfliktlösungsrelevanter Sachverhalt von Bedeutung ist. In den Konfliktsimationen vom Typ des Kaufs/Verkaufs ist es gewöhnlich die Höhe des Preises, in den Kontliktsituationen vom Typ der Lizenznahme/Lizenzvergabe das Lizenzentgelt und in den Kontliktsituationen vom Typ der Fusion/Spaltung die Verteilung der Anteile an der durch Fusion entstehenden neuen wirtschaftlichen Einheit oder an den durch Spaltung neu entstehenden Untemehmungen. Eine mehrdimensionale Konfliktsituation liegt hingegen vor, wenn die Lösung des Konflikts zwischen den Parteien von weiteren Parametern abhängig ist. Konfliktlösungsrelevante Sachverhalte lassen sich in originäre und derivative konfliktlösungsrelevante Sachverhalte unterteilen. ${ }^{19}$ Wirken Parameter unmittelbar entscheidungsfeldverändernd, sind sie den originären konfliktlösungsrelevanten Sachverhalten zuzuordnen. Damit es beispielsweise zur Ảnderung der Rechtsverhältnisse an einer Unternehmung kommt, ist es für die konfligierenden Parteien erforderlich, sich über diese Parameter zu verständigen. Deshalb stehen die originären Sachverhaite zueinander in einem Komplementaritäts- oder Ergänzungsverhältnis. ${ }^{20}$ Unter die derivativen konfliktlösungsrelevanten Sachverhalte fallen diejenigen, die nur mittelbar das Entscheidungsfeld des Bewertungssubjekts verändern. Die derivativen Parameter dienen dazu, die Ausprägungen der originären Sachverhalte herzuleiten oder zu begründen, und stehen somit in einer Mittel-ZweckBeziehung zu diesen originären Verhandlungsgegenständen.

Um eine disjungierte oder unverbundene Konfliktsituation handelt es sich, wenn eine Konfliktpartei das Objekt in einer einzigen Konfliktsituation bewertet, die in keiner Beziehung zu anderen Verhandlungen steht. Gewöhnlich wird in der Literatur nur diese Situation betrachtet. ${ }^{2 t}$ Die jungierte oder verbundene Konfliktsituation liegt hingegen vor, wenn sich das Entscheidungssubjekt zugleich in mehreren Verhandlungssituationen befindet und sich daraus Interdependenzen ergeben. Eine isolierte Betrachtung der einzelnen Konfliktsituationen bildet dabei die Sachlage nicht zutreffend ab..$^{22}$

Mit der Unterscheidung zwischen dominierten und nicht dominierten Konfliktsituationen stellt sich die Frage, ob eine Änderung der Rechtsverhältnisse am Bewertungsobjekt von einer der konfligierenden Parteien beherrscht wird oder nicht. So wird unter einer nicht dominierten Konfliktsituation verstanden, dass keine der Konfliktparteien die Veränderung der Rechtsverhältnisse des Bewertungsobjekts allein, also gegen den Willen und ohne Mitwirkung der anderen Partei, realisieren kann. ${ }^{23}$ Eine dominierte Konfliktsituation liegt dagegen vor, wenn eine der konfligierenden Parteien in der Lage ist, die Rechtsverhältnisse an der zu

\footnotetext{
18 Vgl. hierzu iMATSCHKE (1975), S. 38 If., MATSCHKE (1993).

19 Vgl. MATSCHKE (1975), S. $56 \mathrm{ff}$.

20 Unter die originären kontliktlösungsrelevanten Sachverhaite fallen neben der Höhe des Entgelts z. B. der Umfang der Unternehmung sowie Regelungen über Wettbewerbsverbote. Allein schon die vieifältigen Gestaltungsformen des Entgelts sind den originären Parametem zuzuoránen.

2I Ausnahmen finden sich bisher allein bet MATSCHKE (1975), S. 336 ff., HERING (1999), S. 68 ff. und BROSEL (2002), S. 98 ff., die sich allesamt auch mit jungierten Konfliktsituationen befassen.

22 Vgl. MATSCHKE (1975), S. 34 f.

23 Vgi. MATSCHKE (1979), S. $31 \mathrm{ff}$.
} 
bewertenden Unternehmung auch gegen den erklärten Willen der anderen konfligierenden Partei durchzusetzen."t

Die Problematik der Bewertungssituation sei am Beispiel der UMTS-Versteigerung des Jahres 2000 dargestellt: Vertragsgegenstand und folglich Bewertungsobjekt waren die UMTSLizenzen, also das Recht zum .... Betreiben von Übertragungswegen für das Angebot von Mobilfunkdienstleistungen der dritten Generation (UMTS/LMT-2000) für die Öffentlichkeit im Gebiet der Bundesrepublik Deutschland durch die Lizenznetmerin oder andere." $"$ Im Rahmen der Versteigerung war lediglich das gebotene Lizenzentgelt entscheidungsrelevant. Hinsichtlich der Bedeutung der UMTS-Lizenzen und der Dimension der erreichten Entgelte kann davon ausgegangen werden, dass die Bewertungssubjekte, die Telekommunikationsunternehmungen, zur selben Zeit keinen Erwerbs- oder Veräußerungsverhandlungen durchfiuhrten, die in einer direkten Beziehung zur UMTS-Versteigerung standen oder ähnliche Dimensionen auswiesen. Zwar wurden die Regeln der Versteigerung von staatlicher Seite vorgegeben, es stand aber jeder zugelassenen Telekommunikationsunternehmung frei, sich an der Versteigerung zu beteiligen oder auszusteigen. Insgesamt handelte es sich bei der UMTSVersteigerung aus Sicht der Telekommunikationsunternehmungen um eine eindimensionale, nicht dominierte, disjungierte Konfliktsituation vom Typ des Erwerbs. Vor diesem Hintergrund beschränkt sich der nachfolgend präsentierte Ansatz zur Bewertung von Telekommunikationsunternehmungen und ihren Unternehmungsteilen auf diese Konfliktsituation.

\subsection{Die Konzeptionen der Bewertung}

Im Zentrum der objektiven Unternehmungsbeweriung, die bis in die sechziger Jahre als herrschende Literaturmeinung ${ }^{26}$ galt, stand die Ermittlung eines objektiven Wertes der Unternehmung. Die Bestimmung dieses Wertes sollte entpersonifiziert, also losgelöst von subjektiven Interessen, erfolgen. Der zu ermittelnde Wert haftet der Unternehmung - nach Ansicht der Vertreter dieser Konzeption - an und ist von einem „normalen" Unternehmer realisierbar. ${ }^{27}$ Diese Theorie wollte dem Anspruch genügen, einen unparteiischen Wert zu bestimmen, um Interessengegensätze zwischen konfligierenden Parteien zu überwinden, ohne dabei jedoch Bezug auf die Interessen der Parteien zu nehmen. Aus diesen Gründen orientierten sich die Vertreter dieser Konzeption bei der Ermittlung des objektiven Wertes vornehmlich an vergangenen und gegenwärtigen Verhältnissen. Die Ermittlung des objektiven, substanzorientierten $^{28}$ Wertes liefert auf Grund der Abstraktion vom Bewertungssubjekt und von der jeweiligen Aufgabenstellung keine hinreichende Entscheidungsunterstützung.

24 Vgl. MATSCHKE (1979), S. 33 ff., MATSCAKE (1981), S. 117 t. Zur Unterscheidung in fragmentierte und nicht fragmentierte dominierte Konfliktsituarionen siehe HERING/OCBRICH (2001), S. 23 ff.

Teil A Punkt 1 der UMTS-Musterizenz - Lizenz zum Betreiben von Übertragungswegen für das Angebot von Mobilfunkdienstleistungen der dritten Generation (UMTS/MT-2000) für die Öffentlichkeit im Gebiet der Bundesrepublik Deutschiand.

Siehe z. B. MELLEROWICZ (1952), VIEL (1955), LACKMANN (1962).

Vgi. MELLERONTCZ (1952), S. 60.

38 Zur kritischen Betrachtung des Substanzwertes vgl. MATSCHKE (1990). 
Einen dazu konträren Standpunkt vertraten die Verfechter der subjektiven Unternehmungsbewertungstheorie, deren Anschauung die objektive Unternehmungsbewertungslehre abzulösen suchte..$^{79}$ Die Ermittlung des subjektiven Wertes erfolgt unter Bezugnahme auf die Vorstellungen und Planungen eines konkreten Bewertungsinteressenten. Für jedes Bewertungssubjekt kann die Unternehmung insotern einen grundsätztich verschiedenen, spezifischen Wert haben, der den Grenzpreis der Unternehmung aus Sicht des jeweiligen Bewernungssubjekts widerspiegelt. ${ }^{30}$ Diese Konzeption der Unternehmungsbewertung wird charakterisiert durch die fundamentalen Grundsätze der Bewertung: das Prinzip der Gesamtbewertung, das Prinzip der Zukunftsbezogenheit und das Prinzip der Subjektivität..31

Die kontroversen Anschauungen objektiver und subjektiver Theorie wurden letztlich mit dem Konzept der funktionalen Unternehmungsbewertung ${ }^{\text {j2 }}$ überwunden. Zentraler Punkt der seit Mitte der siebziger Jahre herrschenden Lehre ist die Zweckabhängigkeit ${ }^{33}$ des Unternehmungswertes. Der Wert einer Unternehmung wird mit Bezugnahme auf die Vorstellungen und Planungen des konkreten Bewertungsinteressenten unter expliziter Berücksichtigung der verfolgten Aufgabenstellung der Unternehmungsbewertung ermitteit. „Die Unternehmung hat nicht bloß für jeden Bewertungsinteressenten einen spezifischen Wert, sondern kann auch je nach Aufgabenstellung einen durchaus unterschiedlichen Wert haben." ${ }^{34}$ Die Bewertung erfolgt zweckabhängig; der Unternehmungswert und das Verfahren zu seiner Ermittlung existieren nicht. Die funktionale Bewertungstheorie stützt sich dabei gleichermaßen auf die Grundsätze der Gesamtbewertung, der Zukunftsbezogenheit sowie der Subjektivität, hier ergänzt durch den Grundsatz der Zweckabhängigkeit.

29 Maßgeblichen Anteil am Erfolg der subjektiven Unternehmungsbewertung hatten BUSSE VON COLBE, KiFER und schlieBlich MUNSTERALANN; vgl. BUSSE VON COLBE (1957), MUNSTERMANN (1966) sowie den Nachdnuck der Aufsätze von KAFER (1996).

30 In der Literatur ist die Notwendigkeit des Subjektbezugs im Rahmen von Bewertungen schon durch SCHMALENBACH erkannt worden; vgl. SCAMALENBACH (1917/1918), S. 4.

31 Vgl. :HONSTERMANN (1966), S. 18 ff.

32 Zu den grundlegenden Arbeiten der funktionalen Unternehmungsbewertung, die auch unter der Bezeichnung "Kölner Funktionenlehre" bekannt ist, zählen u. a. MATSCHKE (1969), MATSCHKE (1971), MATSCHKE (1972), MATSCHKE (1975), LATSSCHKE (1976), SIEBEN (1976), MATSCHKE (1979) sowie die Beiträge der ersten Kölner

3 .

$33 \mathrm{Vgl}$. zurn Zweckadăquanzprinzip MOXTER (1983), S. 5 ff.

34 MATSCHKE (1995), S. 973. 


\subsection{Die Funktionen der Bewertung und ihre Wertarten}

Nur, wenn von der jeweiligen Funktion der Bewernung ausgegangen wird, lassen sich die erforderlichen Verfahrensregeln zur Wertermittlung sinnvoll ableiten. ${ }^{35}$ Im Rahmen der Konzeption der funktionalen Bewertung wird in Haupt- und Vebenfunktionen ${ }^{36}$ unterschieden, denen ein Wert dienen kann. Die folgenden Ausführungen beschränken sich auf die Darstellung der Hauptfunktionen, worunter die Entscheidung ${ }^{37}$, die Vermittlung sowie die Argumentation fallen, und der mit ihnen verbundenen Wertarten.

Der Entscheidungswert ist das Ergebnis einer Bewertung im Rahmen der Entscheidungsfunktion. „Der Entscheidungswert zeigt einem Entscheidungssubjekt bei gegebenem Ziel- oder Präferenzsystem und bei gegebenem Entscheidungsfeld an, unter weichen Bedingungen oder unter welchem Komplex von Bedingungen die Durchtührung einer bestimmten vorgesehenen Handlung das ohne diese Handlung erreichbare Niveau der Zielerfullung gerade noch nicht mindert." ${ }^{38}$ Mit anderen Worten gilt der Entscheidungswert als äußerste Grenze der Konzessionsbereitschaft des Entscheidungssubjekts in einer bestimmten Konfliktsituation. Der Entscheidungswert wird durch vier Merkmale ${ }^{39}$ charakterisiert: Er wird hinsichtlich einer definierten Handlung ermittelt (Merknal der Handlungsbezogenheit) und bezieht sich auf ein bestimmtes Entscheidungssubjekt sowie dessen Zielsystem (Merkmal der Subjekt- und Zielsystembezogenheit). Er ist eine kritische Größe (Merkmal des Grenzwertes), die ausschließlich für ein konkretes Entscheidungsfeld und für die darats ableitbaren Alternativen gültig ist (Merkmal der Entscheidungsfeldbezogenheit). ${ }^{40}$

Zielt eine Bewertung auf die Änderung der Rechtsverhältnisse und ist für die Einigung der Konfliktparteien lediglich die Höhe des Kaufpreises oder des Lizenzentgelts von Bedeutung, so entspricht der Entscheidungswert dem Grenzpreis oder dem Grenzentgelt einer Verhandlungspartei in dieser Konfliktsituation. Aus Sicht des präsumtiven Erwerbers ist der Entscheidungswert als Preisobergrenze genau das Entgelt, das er gerade noch zahien kann, ohne durch den Erwerb einen wirtschaftlichen Nachteil hinnehmen zu müssen. ${ }^{+1}$ In Verhandlungssituationen und insbesondere bei Versteigerungen sollte dieses kritische Entgelt zweifelsohne mit Rücksicht auf die Stärke der eigenen Verhandlungsposition ein Wert hinter „vorgehaitener Hand" sein. ${ }^{42}$

35 Vgl. MATSCHKE (1981), S. 115.

36 Ats Nebenfunktionen der Unternehmungsbewertung geiten u. a. die Vertragsgestaltungsfunktion (a), die Steuerbemessungsfunktion (b) und die Kommunikationsfunktion (c). Siehe zu (a) SANFLEBER (1990), SIEBEN/LUTZ (1995), zu (b) SIELAFF (1977), WOXTER (1983), S. 64 ff. sowie zu (c) COENENBERG/SIEBEN (1976), Sp. 4063.

37 HERNG weist darauf hin, dass die Entscheidungstunktion häufig als Beratungsfunktion bezeichnet wird, obwohi der Beratungszweck auch den anderen Funktionen innewohnt; vgi. HERING (1999), S. 3.

38 MATSCHKE (1972), S. 147. Der Entscheidungswert basiert somit auf den investitionstheoretischen Grundsätzen der Zieisetzungs- und Entscheidungsfeldbezogenheit; vgl. zur erforderlichen investitionstheoretischen Fundierung von Bewertungen u. a. HERING (1999), S. 11 ff.

39 Vgi. MATSCHKE (1972), S. 147, MATSCHKE (1975), S. 26.

40 Siehe hierzu auch $S t E B E N / S C H L D B A C H(1994)$, S. 15 ff., insbesondere S. $42 \mathrm{ff}$.

4 Vgl. MATSCHKE (1969), S. 59.

42 Vgl. SIEBEN (1988), S. 86. 
Wird eine Bewertung im Rahmen der Vermittlungsfunktion ${ }^{43}$ durchgefuihrt, ist das Ergebnis der Arbitrium-, Vermittlungs- oder Schiedssprtichwert. Dem Gutachter kommt hierbei die Rolle des unparteiischen Dritten zu. Mit dem Arbitriumwert soll ein für die konfligierenden Parteien zumutbarer Kompromiss hinsichtlich der Bedingungen über die Ändenungen der Rechtsverhältnisse gefunden werden, der die Interessen der Parteien angemessen berücksichtigt. ${ }^{* 4}$ Der Arbitriumwert darf, um als zumutbar zu gelten, die Grenzen der Konzessionsbereitschaft der konfligierenden Parteien nicht verletzen (Grundsatz der Rationalität des Handelns $){ }^{+5}$ Voraussetzung dafür ist die Existenz eines Einigungsbereichs: Der Entscheidungswert ${ }^{46}$ des Erwerbers muss folglich über dem Entscheidungswert des Veräußerers liegen. Gemäß dem Grundsatz der parteienbezogenen Angemessenheit obliegt es dem Gutachter, den Arbitriumwert auf der Grundlage eines gewähiten Gerechtigkeitspostulats innerhalb des so genannten Arbitriumbereichs zu bestimmen. ${ }^{47}$

Das Ergebnis einer Bewertung in der Argumentationsfunktion wird Argumentationswert ${ }^{+8}$ genannt. Der Argumentationswert ist ein parteiischer Wert, dessen Bedeutung in der Beeinflussung des Verhandlungspartners liegt. Mit diesem taktischen Wert soll die eigene Verhandlungsposition verbessert und ein günstigeres Verhandlungsresultat erreicht werden. Argumentationswerte werden zumeist in Form von angeblichen Entscheidungs- oder Arbitriumwerten in den Verhandlungsprozess eingebracht. 49 Zweckdienliche Argumentationswerte setzen sowohl die Kenntnis des eigenen Entscheidungswertes ${ }^{50}$ als auch eine Vermutung über den gegnerischen Entscheidungswert voraus.

t3 Vgl. MATSCHKE (1971), MATSCHKE (1979), MOXTER (1983), S. 22.

t4 Vgi. MATSCHKE (1969), S. 57, MATSCHKE (1984), S. 562.

45 Vgl. MATSCHKE (1979), S. 48 f.

46 Dem Entscheidungswert kommt damit bei der Vermiftlungsfinktion eine zentrale Roile zu.

47 Vgl. MATSCHKE (1971), S. 519, MATSCHKE (1979), S. 112.

48 Siehe MATSCHKE (1976).

49 Die Argumentationsfunktion wird aus Sicht der Wirtschattsprüfer als nicht mit dem Berufsstand vereinbar angesehen. Im Unterschied zur Kölner Funktionenlehre wiró dem Wirschaftsprufer im Rahmen der Unternehmungsbewertung, neben den Funktionen als Berater des Bewertungssubjekts (im Sinne der Entscheidungsfinktion) und als Schiedsgutachter (im Sinne der Vermittlungsfunktion), in erster Linie die Rolie eines neutralen Gutachters zugewiesen, der als zentrale Größe einen „objektivierten Wert" ermitteln soll; vgl. INSTITUT DER WIRTSCHAFTSPRUFER (1983), S. 472 ff. Dieser ,objektiviente“" Wert soll dem Wert der Untemehmung unter der Prämisse der Fortführung des bisherigen Untemehmungskonzepts entsprechen, der im Wesentlichen mangets unzureichender Konkretisierung von noch nicht eingeleiteten zukünttigen Naßnahmen und personenbezogenen Werfaktoren abstrahiert; vgl. SIEPE (1998), S. 5 f. Einwände gegen die statische Orientierung des Modells des „objektivier-

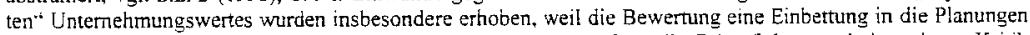
des Bewertungssubjekts verlangt. Aus der Planungsabhängigkeit folgt die Zukunftsbezogenheit; vgl. zur Kritik z. B. LUTZ (1981), S. 151 f., SCHILDBACH (1995). Ein neverlicher Versuch zur Normierung der Verhaltensweisen und Bewertungsverfahren für den Berufsstand wurde inzwischen vorgelegt; vgl. INSTITUT DER WIRTSCHAFTSPRÜFER (2000). Die Aussagefähigkeit des , ,objektivierten“"Wertes wird in Anbetracht der unveränderten Vernachlässigung entscheidender Wertdeterminanten Ansatzpunkt fortwährender Kritik sein; vgl. z. B. HOMMEL BRALN/SCHMOTZ (2001).

50 Auch die Bestimmung eines Argumentationswertes setzi somit die Kenntnis des eigenen Entscheidungswertes voraus. 


\subsection{Die Grundsätze der Bewertung von Telekommunikationsunter- nehmungen und von deren Unternehmungsteilen}

Als die der funktionalen Bewertungstheorie zugunde liegenden bedeutenden Prinzipien ${ }^{\text {il }}$ gelten neben dem Grundsatz der Zweckabhängigkeit die Grundsätze der Gesamtbewertung, der Zukunftsbezogenheit und der Subjektivität. Hinsichtlich des Prinzips der Gesamtbewertung $^{\text {s2 }}$ ist nicht die Summe der Einzelwerte der Vermögensteile dieser Unternehmung oder dieses Unternehmungsteils bewertungsrelevant; vielmehr ist es erforderlich, die im Rahmen der Konfliktsituation zur Disposition stehende Telekommunikationsuntemehmung oder den relevanten Untemehmungsteil als wirtschaftliche Einheit zu betrachten. Im Falle einer isolierten Bewertung der betrieblichen Einzelwerte besteht die Gefahr der Vernachlässigung positiver, aber auch negativer Kombinationseffekte innerhalb des als Einheit zu betrachtenden Bewertungsobjekts, weil die Summe der Einzelwerte nicht mit dem Gesamtwert des Bewertungsobjekts identisch sein muss. ${ }^{53}$ Es ist jedoch nicht ausgeschlossen, dass die Summe der Einzelwerte dem Gesamtwert entspricht. ${ }^{54}$

Das Prinzip der Zukunftsbezogenheit ${ }^{55}$ besagt bezüglich der Bewertung von Telekommunikationsunternehmungen und -unternehmungsteilen, dass für das Bewertungssubjekt nur der Nutzen bewertungsrelevant ist, den ihm das Bewertungsobjekt in der Zukunft stiftet. „Für das Gewesene gibt der Kaufmann nichts. ${ }^{.656}$ Die in der Vergangenheit liegenden Erfolge einer Unternehmung können lediglich als möglicher Indikator zukünftiger Erfolge betrachtet werden. Bei der Bewertung von Produktinnovationen, wie der UMTS-Lizenz, ergibt sich das Problem, dass eine Trendextrapolation auf der Basis der Ergebnisse vorangegangener Perioden überhaupt nicht oder nur sehr eingeschränkt als Prognosehilfe herangezogen werden können. Aus der Zukunftsbezogenheit resultiert das Problem der Unsicherheit ${ }^{57}$, weil dem Bewertungssubjekt im Bewertungszeitpunkt der genaue zukünftige Nutzen des Bewertungsobjekts und alle zukünftigen Handlungsaltemativen und -konsequenzen nicht bekannt sind.

Die Bestimmung des Wertes von Telekommunikationsunternehmungen oder ihren Unternehmungsteilen erfordert gemäß dern Prinzip der Subjektivitä $t^{58}$ die Einbettung des Bewertungsobjekts in die Planungen des Bewertungssubjekts. ${ }^{59}$ Signifikant ist somit die Zielsystem-, Entscheidungsfeld- und Handlungsbezogenheit des Wertes. Dementsprechend ist der Wert durch die vom Bewertungssubjekt verfolgten Ziele, durch die aus dem Entscheidungsfeld verfugbaren finanz- und realwirtschaftlichen Handlungsmöglichkeiten und beschränkungen sowie durch die von der Unternehmung für das Bewertungsobjekt geplante Verwendung determiniert. Ferner ist Ausfluss des Subjektivitätsprinzips, dass die vom Bewertungs-

51

54

55

56

57

58

59

\footnotetext{
Vgi. zu diesen Ausführungen MATSCHKE/BRÖSEL (2003).

Vgl. zum Prinzip der Gesamtbewertung, das in der Literatur auch ais Prinzip der Bewertungseinheit zu finden ist, AULER (1926/1927), S. 42, BALLWIESER/LEUTHIER (1986), S. 548.

V Gl. MUNSTERMANN (1966), S. 18.

Dies ist möglich, wenn z. B. eine sofortige Weiterveräußerung der Untemehmung geplant ist.

Vgl. auch BALLWIESER/LEUTHIER (1986), S. 548.

MUNSTERMANN (1966), S. 21.

Vgl. auch KEUPER (2002), S. $458 \mathrm{f}$.

Vgl. aLch MOXTER (1983), S. 23.

Der Subjektbezug, der das Fundament der modemen Bewertungstheorie der Köiner und Greifswalder Schule darstellt, bleibt bisher in der angelsächsischen Bewertungsiehre weitestgehend unberücksichtigt; vgl. OLBR/CH
$(2000)$, S. $458 \mathrm{f}$.
} 
subjekt erwarteten individuellen positiven und negativen Verbundeffekte bei der Wertermittlung berücksichtigt werden müssen. Auf Grund unterschiedlicher Planungen, Synergiepotenziale $^{\dot{0} 0}$ sowie Verwertungsmöglichkeiten und -beschränkungen ${ }^{\circ 1}$ haben Telekommunikationsunternehmungen und ihre Unternehmungsteile für jedes Bewertungssubjekt einen individuellen Wert. Aus identischen Nutzenerwartungen verschiedener Bewertungssubjekte folgt keinesfalls ein identischer Wert des Bewertungsobjekts, wenn z. B. allein die den Subjekten alternativ zur Verfügung stehenden Kapitalverwendungsmöglichkeiten differieren. ${ }^{62}$

Die Abgrenzung der Zukunftserfolge

\subsection{Die grundlegenden Prinzipien zur Abgrenzung der Zukunftserfolge}

Für den präsumtiven Erwerber sind im Rahmen der Bewertung alle durch das Bewertungsobjekt hervorgerufenen künftigen Erfolge von Bedeutung. Das Bewertungsobjekt stiftet dem Bewertungssubjekt im Falle des Erwerbs einen künftigen Nutzen und trägt damit zu dessen Zielerfüllung bei. Die Ermittlung dieser für das Bewertungssubjekt relevanten Erfolge aus dem Bewertungsobjekt steht nicht im Mittelpunkt der Bewertungstheorie. Abgrenzung und Quantifizierung des durch die zu bewertenden Telekommunikationsunternehmungen gestifteten Nutzens obliegen vielmehr den Fachleuten der Telekommunikationsbranche. Die Gewinnung der Zukunftserfolge, wechselseitige Abhängigkeiten und die Veränderungen der Zukunftserfolge während des im Rahmen der Bewertung betrachteten Zeitraumes werden gewöhnlich in der Literatur vernachlässigt..$^{63}$ Die Qualität eines durch bestimmte Modelle ermittelten Wertes für die Telekommunikationsunternehmung wird jedoch determiniert durch die Qualität der Informationen sowie der abgegrenzten und quantifizierten künftigen Erfolge, die für die Bewertung zur Verfügung gestellt werden. Durch die in der Telekommunikationsbranche gegebenen hohen Risiken steigen die Anforderungen an die Prognose zukünftiger Erfolgsströme. Sollen mit der Bewertung sinnvolle Ergebnisse erzieit werden, ist der aus der Unternehmung resultierende Erfolg zweckentsprechend abzugrenzen und zu quantifizieren. Vor diesem Hintergrund werden nunmehr mit dem Gesamtertrags-, dem Zufluss- und dem Verbundberücksichtigungsprinzip die wesentlichen Grundsätze dargestellt, die bei der Abgrenzung der durch das Bewertungsobjekt induzierten künftigen Erfolge zu beachten sind. ${ }^{64}$

Prinzipiell ist für den präsumtiven Erwerber gemäß dem Gesamtertragsprinzip ${ }^{63}$ unter dem künftigen Erfolg die Summe aller Vorteile zu verstehen. die ihm infolge des Erwerbs der Telekommunikationsunternehmung oder von dessen Unternehmungsteilen zuflössen. Unter die-

\footnotetext{
60 Siehe zu Synergie- oder Verbundeffekten MOXTER (1983), S. 91 ff.. WEBER (1991), OLBRICH (1999), S. $20 \mathrm{ff}$.

6) Hierunter tallen z. B. der Bestand an eigenen Vertragskunden und das vorhandene Festnetz.

62 Vgl. BALLIVIESER/LEUTHIER (1986), S. 549.

63 Siehe hierzu auch DECHANT/TROST (2001), S. 234.

64 Vgl. zu den folgenden Ausfïhrungen MOXTER (1983), S. 75 ff. und S. $91 \mathrm{ff}$.

65 Vgl. hierzu MOXTER (1983), S. 75 ff.
} 
se Vorteile fallen sowohl finanzielle als auch uichtfinanzielle Elemente. Neben zu verzeichnenden Einzahlungsüberschüssen kann beispielsweise der Erwerb einer Telekommunikationsunternehmung den Bekanntheitsgrad und das Ansehen eines Bewertungssubjekts beeinflussen. Auf Grund mangelnder Quantifizierungsmöglichkeiten erweist sich die Beurteilung der nichtfinanziellen Vorteile als besonders schwierig. Ausgehend vom individuellen Zielsystem des Bewertungssubjekts ist es erforderlich, alle interessierenden Sachverhalte zu identifizieren und deren Gewichtung zu bestimmen. ${ }^{66}$ Im vorliegenden Falle wird davon ausgegangen, dass sich das Interesse der Bewertungssubjekte vornehmlich auf tinanzielle Vorteile oder auf einen finanziellen Nutzen richtet, das heißt, Bewertungssubjekte streben nach einem Zufluss, der in Form von Zahlungen an die jeweiligen Eigner der bewertenden Unternehmung (Entnahmen oder Ausschüttungen) sowie Auszahlungsersparnissen der Eigner auftreten und gemessen werden kann. Hinsichtlich dieser Entnahmezielsetzung sollen im Rahmen der Ausführungen allein die monetären Vorteile ais bewertungsrelevant gelten und zur Ermittlung des Entscheidungswertes herangezogen werden. Diese vereinfachte Annahme darf aber keinesfalls dazu führen, nichtfinanzielle Vorteile zu vernachlässigen oder unberücksichtigt zu lassen. Vielmehr sollen durch den Entscheidungsträger neben dem Entscheidungswert, in dem lediglich tinanzielle Vorteile berücksichtigt werden, auch die nichtfinanziellen Vorteile bei der Preisbestimmung abgewogen werden, denn grundsätzlich besteht der Zukunftserfolg aus den gesamten Vorteilserwartungen.67

Maßgeblich für die Bewertung von Telekommunikationsunternehmungen ist entsprechend dem Zuflussprinzip ${ }^{68}$ der Vorteilsstrom, der dem präsumtiven Erwerber mit dem Erwerb des Bewertungsobjekts zufließt. Die Eigner der bewertenden Unternehmung sind gemäß der eriäuterten Entnahmezielsetzung an einem finanziellen Zufluss in Form einer Entnahme, Auszahlung oder Ausschüttung interessiert, der ihnen zur Befriedigung ihrer Konsumwünsche zur Verfïgung steht. Hierunter fallen auch Auszahlungsersparnisse, die durch Leistungen der Untemehmung an ihre Eigner hervorgerufen werden.

Der Einfluss des Bewertungsobjekts auf die Bedürfnisbefriedigung der Eigentümer ist über die dadurch ausgelösten Zahlungskonsequenzen messbar. ${ }^{69}$ Als Rechengröße zur Beurteilung des künftigen Nutzens des Bewertungsobjekts dienen somit Zahiungsgrößen. Ein- und Auszahlungen sind objektiv nachprüfbar, weil sie weder bilanziellen Bewertungseinflüssen noch Periodisierungsüberlegungen unterliegen. Die Beschränkung auf Geldzu- und -abflüsse als relevante finanzielle Größen vermeidet die Gefahr von Doppelzählungen. ${ }^{70} \mathrm{Als}$ ZahlungsgröBen kommen sowohl Einzahlungsüberschüsse als auch Auszahlungsersparnisse in Betracht. Erfolgsgrößen haben dabei nur einen Einfluss auf die Bewertung, wenn sie die Höhe der Zahlungen beeinflussen." Die zu bewertenden Telekommunikationsuniemehmung oder dessen zu bewertende Unternehmungsteile werden im Rahmen der Bewertung als ein unsicherer künftiger Zahlungsstrom gedeutet. Der relevante Zahlungsstrom, der dem Bewertungsobjekt

66 Siehe hierzu das Erfolgsermittlungsmodell in MATSCHKE (1975), S. 75 ff.

67 Vgl. auch SERFLING/PAPE (1995), S. 815.

68 Vgl. zum Zuflussprinzip MOXTER (1983), S. 79 ff.

69 Dieser Zusammenhang ergibt sich, weil der Kapitalwert der Ausschüttungen an die Eigner einer Unternehmung genau der Summe der Kapitalwerte aller vorteilhaften Investitions- und Finanzierungsobjekte entspricht; vgl. HERNG (1999), S. 17 f.

$70 \mathrm{Vgl}$. hierzu das Beispiel von MOXTER (I983), S. 79 f.

71 Die Höhe der Zahiungsgrößen wird z. B. beeinflusst durch erfolgsabhängige Steuerzahlungen; vgl. auch HERING (1995), S. 9. 
zuzurechnen ist, ergibt sich somit aus den kontinuierlich oder diskontinuierlichen auftretenden Einzahlungen und Auszahlungen.?

Treten beim Erwerb einer Telekommunikationsunternehmung oder von dessen Untemehmungsteilen Eingliederungseffekte auf, sind diese bei der Ermittlung des Zukunftserfolgs zu beachten. Der bewertungsrelevante Vorteilsstrom im Sinne der (Ein-)Zahlungsüberschüsse ZÜ ergibt sich in der Periode oder zum Zeitpunkt $t$ somit gemäß dem Verbundberïcksichtigungsprinzip $p^{\gamma 3}$ aus der Differenz der zu verzeichnenden Erfolge der bewertenden Unternehmung mit $\left(Z \mathrm{U}^{\mathrm{mBO}}\right)$ und ohne $\left(\mathrm{Z} \mathrm{U}^{\mathrm{mBO}}\right)$ dem Bewertungsobjekt:

$$
Z \ddot{U}_{i}=Z \ddot{U}_{t}^{m B O}-Z \ddot{U}^{\mathrm{oBO}} \text {. }
$$

Da sich die Zahlungsüberschüsse jeweils als Differenz zwischen den Einzahlungen (inklusive der Auszahlungsersparnisse) E und Auszahlungen A ergeben, resultiert hieraus:

$$
\begin{aligned}
& Z \ddot{U}_{t}=\left(E_{t}^{\mathrm{mBO}}-A_{t}^{\mathrm{mBO}}\right)-\left(E_{t}^{\mathrm{OBO}}-A_{t}^{O B O}\right) \text { sowie } \\
& Z \ddot{U}_{t}=E_{t}^{m B O}-E_{t}^{0 B O}-A_{t}^{\mathrm{mBO}}+A_{t}^{\circ B O} .
\end{aligned}
$$

Zur Ermittlung der bewertungsrelevanten Zahlungsüberschüsse ist demnach die Ermittlung der Einzahlungen der bewertenden Unternehmung mit $\left(\mathrm{E}_{\mathrm{i}}^{\mathrm{mBO}}\right)$ und ohne $\left(\mathrm{E}_{\mathrm{t}}^{\mathrm{BOO}}\right)$ dem Bewertungsobjekt sowie der Auszahlungen der bewertenden Unternehmung mit $\left(A_{t}^{m B O}\right.$ ) und ohne $\left(A_{t}^{\circ B O}\right)$ dem Bewertungsobjekt erforderlich.

In der Tefekommunikationsbranche können Verbundeffekte z. B. in Form von Auszahlungsersparnissen bei der bewertenden Unternehmung in Anbetracht ansonsten erforderlicher Netzerweiterungen oder -optimierungen sowie Kundenmanagement- und Kundenabrechnungssysteme auftreten. Insbesondere beim Agieren in neuen Märkten lässt sich durch Unternehmungswachstum auf Grund einer Akquisition oft eine herausragende Stellung gegenüber den Konkurrenten erreichen; unter Umständen mit der Konsequenz einer mehr ais nur additiven Verknüpfung der beiden unternehmungsbezogenen Marktanteile. Kostenseitig kann sich beispielsweise eine Fixkostendegression durch größere Produktionsmengen ergeben. Darüber hinaus kann es auch zu negativen Verbundeffekten, wie z. B. ,doppeltes Personal“", kommen. Da die jeweiligen Verbundeffekte durch die Synthese von der erwerbenden und der zu erwerbenden Unternehmung generiert werden, spiegelt das Verbundberücksichtigungsprinzip sowohl das Prinzip der Gesamtbewertung als auch das Prinzip der Subjektivität wider. Neben diesem zu beachtenden Problem der Erfolgsabhängigkeit ist das Verbundberücksichtigungsprinzip außerdem im Falle einer jungierten Konfliktsituation relevant. Liegt eine solche verbundene Konfliktsituation vor, muss folglich berücksichtigt werden, dass der Entscheidungswert der einen Unternehmung jeweils in Abhängigkeit des Verhandlungsergebnisses um die andere Untemehmung zu bestimmen ist. ${ }^{74}$

\footnotetext{
72 Zur Vereinfachung wird in der Untemehmungsbewertung ebenso wie in der Investitionsrechnung ein hypothetischer diskontinuierlicher Zahiungsstrom untersteilt; vgl. auch MATSCIKE (1993), S. 58.

73 Siehe MOXTER (1983), S. 91 ff., der statt vom Exfolg vom Ertrag spricht.

$74 \mathrm{Vgl}$. MATSCHKE (1975), S. $336 \mathrm{ff}$.
} 


\subsection{Ein Ansatz zur Prognose der Zukunftserfolge}

\subsubsection{Die Prognose der Einzahlungen}

Die sorgfältige ${ }^{75}$ Abgrenzung und Quantifizierung der relevanten Zahlungen durch die in der Telekommunikationsbranche agierenden Fachkräfte erfordern die strikte Einhaitung der dargestellten Prinzipien. Nachfolgend wird ein für Telekommunikationsuntemehmungen entwickelter heuristischer Ansatz zur Unterstützung der Prognose zukünftiger Erfolgsströme dargestellt, welches in einer führenden deutschen Telekommunikationsunternehmung eingesetzt wird. ${ }^{76}$ Dieser Prognoseansatz gliedert sich im Hinblick auf die dargestellte Formel zur Ermittlung der bewertungsrelevanten Zahlungsüberschüsse $Z \ddot{U}_{t}$ in die Bestandteile „Einzahlungsprognose" mit einem Markt- und Umsatzmodell (zur Ermittlung der jeweiligen $E_{t}^{\text {mBO }}$ und $\mathrm{E}_{\mathrm{t}}^{\mathrm{OBO}}$ ) sowie "Auszahlungsprognose" mit einem Investitions- und Kostenmodell (zur Ermittlung der jeweiligen $A_{t}^{\mathrm{mBO}}$ und $A_{t}^{\circ 0}$ ). Mit diesem Ansatz soll nicht nur die fundierte Prognose der Zahlungsüberschüsse unterstützt werden; der Ansatz ermöglicht zudem eine größere Transparenz der Schätzungen und lässt die Verfahren zur Berücksichtigung der Unsicherheit eine größere Aussagekraft gewinnen, weil die Wirkungen bestimmter Parameterkonstellationen untersucht und transparent aufgezeigt werden können.

Die Bewertung von Unternehmungen mit großen Investitionen in den UMTS-Standard muss vor allem an den Produkten ansetzen, wobei insbesondere die durch den UMTS-Standard hervorgerufenen innovativen Produkte zu berücksichtigen sind. Die Prognose der Zukunftserfolge wird am Fall einer Produktinnovation demonstriert, weil diese auf Grund der hohen Kapitalbindung über einen langen Zeitraum und der vielen Unwägbarkeiten besonders sorgfältig zu erfolgen hat. Einen wesentlichen Schwerpunkt dieser Ausführungen wird die mögliche Datengewinnung bilden. ${ }^{77}$ Die Prognose der Einzahlungen erfordert die Ermittlung der möglichen Absatzmengen - hier identisch mit den Kundenzahlen - und der Produktpreise, welche wiederum die Basis zur Schätzung der Umsatzgrößen darstellen. Der Ansatz zur Einzahlungsprognose wird in die folgenden zehn Schritte unterteilt:

\section{Schritt 1: Segmentierung}

Im ersten Schritt erfolgt die Segmentierung des Marktes. ${ }^{78}$ Dabei sind zuerst die einzelnen Kundensegmente s ( $\mathrm{z}$. B. Kundensegmente Haushalte und Geschäftskunden) zu bestimmen. Anschließend erfolgt für jedes Kundensegment die Schätzung der Größe des Segments $S_{t}^{s}$ sowie der bedürfnisgerechten Produktvarianten, wie z. B. unterschiedliche Bandbreiten. Hinsichtlich der Bestimmung des jeweiligen Kundensegmentumfangs $S_{t}^{s}$ kann $z$. B. auf das Statistische Bundesamt als Quelle zurückgegriffen werden. Für die Entwicklung von Produktvarianten sind, über die Analyse der technischen Machbarkeit hinaus, Verbraucherbefragungen durchzuführen.

\footnotetext{
75 Siehe weiterführend die Ausführungen zur Sorgfaltsprüfung in OLBR/CH (2002), S. 695 ff. Nachfolgende Darstellungen zum Prognosemodell erfolgen in enger Anlel.nung an DECHAvT/TROST (2001). Zur praktischen Anwendung siehe zudem DECHANT/BRASSLER (2003).

77 Einzelheiten der technischen Realisienung bleiben hier unberücksichtigt.

7 Der Index $t$ bezeichnet die jeweilige Periode als Zeitraum zwischen den Zeitpunkten $\mathrm{-} \mathrm{I} \mathrm{und} t$ (hier Jahr) oder den jeweiligen Zeitpunkt (z. B. bezieht sich die Bestandsgröße „Kunden“ auf das Jahresende).
} 


\begin{tabular}{|l|c|c|}
\hline Bezeichnung & Variable/Formel & Art der Ermittlung \\
\hline Bestimmung der Kundensegmente & $\mathrm{S}$ & $\begin{array}{c}\text { Unternehraungs- } \\
\text { entscheidung }\end{array}$ \\
\hline $\begin{array}{l}\text { Umfang der jeweiligen Kunden- } \\
\text { segmente }\end{array}$ & $\mathrm{S}_{\mathrm{t}}^{\text {s }}$ & Schätzung \\
\hline Produktvarianten & Index h & Analyse \\
\hline
\end{tabular}

Tabelle l: $\quad$ Kundensegmentierung

\section{Schritt 2: Interdependente Schätzung vom potentiellen Marktumfang und von möglichen Absatzpreisen}

In einem zweiten Schritt werden der potentielle Marktumfang und die möglichen Absatzpreise für die jeweiligen Segmente s durch eine interdependente Schätzung ermitteit. Zur Vereinfachung wird hier auf die mengenseitige Berücksichtigung der Kundenverteilung auf die Produktvarianten verzichtet, was der Betrachtung eines „Durchschnittskunden“" entspricht. Diese Kundenverteilung wird erst zu einem späteren Zeitpunkt (Schritt 6 und 7) in die Preisund Erlöskalkulationen einbezogen.

Die Schätzung von potentiellen Penetrationsraten $\mathrm{PP}_{\mathrm{t}}^{\mathrm{s}}$ ist bei Produktinnovationen meist schwierig, weil neben der vermeintlichen Nachfrage u. a. auch die technischen Restriktionen (z. B. Engpässe bei den Zulieferem von Equipment) und die aus unternehmerischen Entscheidungen resultierenden Einschränkungen berücksichtigt werden müssen. Häufig ist es sogar notwendig, mehrere multiplikativ verknüpfte Penetrationsraten zu modellieren. Aktueile sowie vergangene Penetrationsraten können z. B. einschlägigen Fachzeitschriften entnommen oder durch Spezialstudien ermittelt werden. Zudem stellt die Gesellschaft für Konsumforschung auf Anfrage detailliertes Datenmaterial zur Verfügung und gibt einen mithilfe von Befragungen ermittelten Prognosewert für das folgende Jahr an. Es ist zu beachten, dass diese Daten jedoch nur sehr beschränkt Aufschluss über mehr als ein Jahr in der Zukunft liegende Penetrationsraten geben. Aus unterschiedlichen Quellen stammende Penetrationsraten weichen häufig erheblich voneinander ab. Sogar Spezialstudien geben teilweise keine klare Abgrenzung der Marktsegmente und lassen offen, ob technische oder weitere Restriktionen bereits berücksichtigt sind. Auch bei der Übertragung von Penetrationsraten von „Vorreiterländem* auf den betrachteten Markt ist hinsichtlich unterschiedlicher Gewohnheiten und Spezifika höchste Vorsicht geboten. Vor diesem Hintergrund ist die originäre Schätzung als Methode zu präferieren. ${ }^{79}$ Jede Penetrationsrate ist über eine Identifikation und Ranglozierung der Produkttreiber (z. B. monatliche Nutzungszeit, Geschwindigkeit und Komfort der Datenübertragung, hochbitratige Anwendungen, Status, Preis) und ihrer Ausprägung in dem nach Zielgruppen (z. B. Studenten, Singles mit hober Bildung Familien mit hohem Einkommen, Kinder in höheren Schulen) kategorisierten Segment abzuschätzen.

Penetrationsraten sind in enger Abstimmung mit den erwarteten Preisen zu bemessen. ${ }^{80}$ Bei der hierzu notwendigen gewissenhaften Analyse der Kundenbedürfnisse steht die Frage im

79

30

An dieser Stelle sei auf den Prozess der Marktforschung verwiesen; siehe KOPPELLANN (1997), S. 114 ff.

Dargestellt am Beispiel UMTS ist anschließend fur jede Zieigruppe unter Gewichtung der Treiber eine Penetrationsrate UMTS und zwar ausgehend von der zielgruppenspezifischen Penetrationsrate Mobilfunk zu schätzen.

Unter Einbeziehung der jeweiligen Größe der Ziełgruppe und der Penetrationsrate Mobilfunk sowie der Anzah! 
Mittelpunkt: ,Welche Zielgruppen haben welchen Nutzen und wie hoch ist dafür deren Zahlungsbereitschaft?" Insbesondere für die Schätzung der Marktpreise $p_{h . s}^{s . M}$ und $g_{h . t}^{s, M}$ sind Verbraucherbefragungen eine unverzichtbare Informationsquelle. Der verwendete Index $M$ macht deutlich, dass es sich - in Abgrenzung zu den nachfolgend verwendeten unternehmungsspezifischen Größen (Index U) - um marktspezifische Größen handelt.

\begin{tabular}{|l|c|c|}
\hline Bezeichnung & Variable/Formel & Art der Ermittlung \\
\hline potentielle Penetrationsraten & $\mathrm{PP}_{\mathrm{t}}^{\mathrm{s}} \in[0 ; 1]$ & Schätzung \\
\hline segmentspezifische Absatzpreise & $\mathrm{p}_{\mathrm{h}, \mathrm{l}}^{\mathrm{s}, \mathrm{M}}$ & Schätzung \\
- Monatsgebühren & $\mathrm{g}_{\mathrm{h}, \mathrm{h}}^{\mathrm{s}, \mathrm{h}}$ & Schätzung \\
- einmalige Anschlussgebühren & & \\
\hline
\end{tabular}

Tabelle 2: $\quad$ Interdependente Schätzung vom potentiellen Marktumfang und von möglichen Absatzpreisen

Schritt 3: $\quad$ Ermittlung der potentiell adressierbaren Kundensegmente

Die Ermittlung der potentiell adressierbaren Kundensegmente erfolgt nunmehr im dritten Schritt durch die Multiplikation der jeweiligen Kundensegmente $S_{t}^{s}$ mit den korrespondierenden potentiellen Penetrationsraten $\mathrm{PP}_{\mathrm{t}}^{s}$.

\begin{tabular}{|l|c|c|}
\hline Bezeichnung & Variable/Formel & Art der Ermittlung \\
\hline $\begin{array}{l}\text { Anzahl der potentiell adressierba- } \\
\text { ren Kunden pro Segment }\end{array}$ & $\mathrm{S}_{\mathrm{t}}^{\mathrm{s}} \cdot \mathrm{PP}_{\mathrm{t}}^{\mathrm{s}}$ & Berechnung \\
\hline
\end{tabular}

Tabelle 3: $\quad$ Ermittlung der potentiell adressierbaren Kundensegmente

\section{Schritt 4: Festlegung eines Tarif- und Angebotmodells}

Es sind strategische Entscheidungen der Unternehmung, ob der Gesamtmarkt oder nut Teilmärkte adressiert und wie schnell die Adressatenkreise erweitert werden sollen. Der Adressierungsgrad wird formal über einen so genannten segmentspezifischen Potenzialausschöpfungskoeffizienten $\alpha_{t}^{z, U}$ mit $\alpha_{t}^{\mathrm{s}, \mathrm{U}} \in[0 ; 1]$ erfasst. Insbesondere für auf die Festnetzkommunikation fokussierte Unternehmungen ist diese Entscheidung von weitreichender Bedeutung. Als Einflussgrößen kommen u. a. das Leitbild der Untemehmung, die Liefer- und Produktionszeiten, die Risiko- und Kooperationsbereitschaft, die Lukrativität der regionalen Marktpotenziale, die Investitionshöhe sowie die Finanzlage einschließlich des Verschuldungspotenzials der Unternehmung in Frage. Hauptsächlich in den Anfangsjahren der Marktdurchdringung werden die Erfahrungen gemacht, dass ein schneller Einstieg in den flächendeckenden Massenmarkt häufig die Unternehmungsprozesse überfordert, was sich u. a. in einem schlechten Service äußerte. Die Anzahl der pro Segment adressierbaren Kunden ergibt sich schließlich aus der Größe des Kundensegments $\mathrm{S}_{!}^{\mathrm{s}}$, der Penetrationsrate $\mathrm{PP}_{t}^{\mathrm{s}}$ und dem Potenzialausschöpfungskoeffizienten $\alpha_{i}^{\text {s.U }}$.

der Haushalte sind dann die zielgruppenspezifischen Penetrationsraten UMTS in eine durchschnittliche Penetrationsrate UMTS zu überführen. 
Darüber hinaus ist im Rahmen des Tarif- und Angebotsmodells die preisliche Positionierung - hier dargestellt über segmentspezifische Preisanpassungskoeffizienten $\left(\mathrm{q}_{h, \mathrm{~s}}^{\mathrm{s} . \mathrm{j}}\right)$ - gegenüber den Wettbewerbern festzulegen. ${ }^{31}$ In der praktischen Anwendung sind Kalkulationen für verschiedene solcher Tarif- und Angebotsmodelle durchzuführen, wobei die Dynamik der Marktpreise in Abhängigkeit von der Preisentscheidung der Unternehmung zu berücksichtigen ist. Aus den im Schritt 2 geschätzten Marktpreisen $\mathrm{p}_{\mathrm{h}, \mathrm{t}}^{\mathrm{s}, \mathrm{M}}$ und $\mathrm{g}_{h, \mathrm{t}}^{\mathrm{s}, \mathrm{M}}$ lassen sich über einen unternehmungsspezifischen Preisanpassungskoeffizienten $q_{h, s}^{j, U}$ die entsprechenden produktsegmentspezifischen Monatspreise $\mathrm{p}_{\mathrm{h}, \mathrm{L}}^{\mathrm{s}, \mathrm{L}}$ und Anschlussgebühren $\mathrm{g}_{\mathrm{h}, \mathrm{t}}^{\mathrm{i}, \mathrm{U}}$ ableiten.

\begin{tabular}{|c|c|c|}
\hline Bezeichnung & Variable/Formel & Art der Ermittlung \\
\hline $\begin{array}{l}\text { Potenzialausschöpfungskoeffi- } \\
\text { zient, so dass: }\end{array}$ & $\alpha_{i}^{j_{i} U}$ & $\begin{array}{l}\text { Untemehmungs- } \\
\text { entscheidung }\end{array}$ \\
\hline $\begin{array}{l}\text { Anzahl der adressierbaren } \\
\text { Kunden }=\end{array}$ & $\mathrm{S}_{\mathrm{t}}^{s} \cdot \mathrm{PP}_{\mathrm{t}}^{\mathrm{s}} \cdot \alpha_{\mathrm{t}}^{\mathrm{s}, \mathrm{U}}$ & Berechnung \\
\hline $\begin{array}{l}\text { Preisanpassungskoeffizient, } \\
\text { so dass: } \\
\text { produktsegmentspezifische } \\
\text { Monatspreise = } \\
\text { produktsegmentspezifische An- } \\
\text { schlussgebühren = }\end{array}$ & $\begin{aligned} & q_{h, t}^{s, U} \\
p_{h, t}^{s, U}= & q_{h, t}^{s, U} \cdot p_{h, t}^{s, M} \\
g_{h, t}^{s, U}= & q_{h, t}^{s, U} \cdot g_{h, t}^{s, M}\end{aligned}$ & $\begin{array}{l}\text { Unternehmungs- } \\
\text { entscheidung } \\
\text { Berechnung } \\
\text { Berechnung }\end{array}$ \\
\hline
\end{tabular}

Tabelle 4: $\quad$ Festlegung eines Tarif- und Angebotmodells

\section{Schritt 5: $\quad$ Schätzung des Marktanteils und Berechnung der Kundenzahlen}

Die Schätzung des Marktanteils der Unternehmung im jeweiligen (Teil-)Segment MA MA $_{t}^{\text {.U }}$ erfolgt im Schritt 5 unter Rückgriff auf die im Schritt 4 anvisierten Potenziale. Die Ermittlung des von der bereits festgelegten Tarifgestaltung abhängigen potentiellen Marktanteils sollte auf strategische Analysen ${ }^{82}$ der Unternehmung und der wesentlichen Wettbewerber der reievanten strategischen Gruppe fundieren. Analyseobjekte sind u. a. die bisherigen Marktanteile, Produktstrategie und -portfolio, Vertriebs- und Produktionskapazität sowie Unternehmungskooperationen. Anschließend kömnen die segmentspezifischen Kundenzahlen (gleichbedeutend mit der Produktmenge) der Unternehmung $m_{:}^{\text {s.U }}$ aus der Größe des Kundensegments $S_{t}^{s}$, der Penetrationsrate $P P_{t}^{s}$, dem Potenzialausschöpfungskoeffizienten $\alpha_{t}^{\text {s.U }}$ und diesem Marktanteil $\mathrm{MA}_{:}^{\mathrm{s}, U}$ berechnet werden.

\begin{tabular}{|l|c|c|}
\hline Bezeichnung & Variable/Formel & Art der Ermittlung \\
\hline Marktanteil & $\mathrm{MA}_{\mathrm{s}}^{\mathrm{s}, \mathrm{U}}$ & Schätzung \\
\hline Kundenzahl & $\mathrm{m}_{\mathrm{s}}^{\mathrm{s}, \mathrm{u}}=\mathrm{S}_{\mathrm{s}}^{\mathrm{s}} \cdot \mathrm{PP}_{\mathrm{s}}^{\mathrm{s}} \cdot \alpha_{\mathrm{s}}^{\mathrm{s} U} \cdot \mathrm{MA}_{\mathrm{s}}^{\mathrm{s}, \mathrm{U}}$ & Berechnung \\
\hline
\end{tabular}

Tabelle 5: $\quad$ Schätzung des Marktanteils und Berechnung der Kundenzahlen

8. Vgl. KOTLER/BLIEMEL (2001), S. $114 \mathrm{ff}$.

82 Hienunter tallen z. B. die Stärken- und Schwächen- sowie die Potenzialanalyse. 


\section{Schritt 6: Schätzung der Nachfrageverteilung}

Nunmehr erfolgt im sechsten Schritt die Schätzung der bislang vemachlässigten Verteilung der Nachfrage (nachfolgend als Nachfrageverteilung bezeichnet) $\beta_{h s}^{\text {s.U }}$ auf die unterschiedlichen Produktvarianten der jeweiligen Segmente.

\begin{tabular}{|l|c|c|}
\hline Bezeichnung & Variable/Formel & Art der Ermittlung \\
\hline $\begin{array}{l}\text { unternehmungsspezifische Nach- } \\
\text { frageverteilung }\end{array}$ & $\beta_{h, s}^{s, \mathrm{~J}}$ mit $0 \leq \beta_{h, s}^{\mathrm{s}, \mathrm{U}} \leq 1, \sum_{h} \beta_{h, \mathrm{~L}}^{\mathrm{s}, \mathrm{U}=\mathrm{L}}$ & Schätzung \\
\hline
\end{tabular}

Tabelle 6: $\quad$ Schätzung der Nachifrageverteilung

\section{Schritt 7: $\quad$ Berechnung der (Durchschnitts-)Preise}

Im Anschluss an die Ermittlung der Nachfrageverteilung $\beta_{\mathrm{h} \perp}^{\mathrm{s}, \mathrm{U}}$ kann mit dieser und den produktvariantenspezifischen Monatspreisen $p_{h, t}^{s, u}$ sowie den Anschlussgebühren $g_{h, u}^{s, u}$ der durchschnittliche Monatspreis $p_{\text {: }}^{s, U}$ sowie die durchschnittliche Anschlussgebühr $g_{t}, \mathrm{~s}$ berechnet werden, welche ebenfalls als unternehmungsspezifische Durchschnittspreise zu charakterisieren sind.

\begin{tabular}{|l|c|c|}
\hline Bezeichnung & Variable/Formel & Art der Ermittlung \\
\hline $\begin{array}{l}\text { unternehmungsspezifischer durch- } \\
\text { schnittlicher Monatspreis }\end{array}$ & $\mathrm{p}_{\mathrm{i}}^{\mathrm{s}, \mathrm{U}}=\sum_{\mathrm{i}} \beta_{\mathrm{h}, \mathrm{f}}^{\mathrm{s}, \mathrm{U}} \cdot \mathrm{p}_{\mathrm{h}, \mathrm{s}}^{\mathrm{s}, \mathrm{U}}$ & Berechnung \\
$\begin{array}{l}\text { unternehmungsspezifische durch- } \\
\text { schnittliche Anschlussgebühr }\end{array}$ & $\mathrm{g}_{\mathrm{i}}^{\mathrm{s}, \mathrm{U}}=\sum_{\mathrm{i}} \beta_{\mathrm{h}, \mathrm{t}}^{\mathrm{s}, \mathrm{U}} \cdot \mathrm{g}_{\mathrm{t}, \mathrm{s}}^{\mathrm{s}, \mathrm{U}}$ & Berechnung \\
\hline
\end{tabular}

Tabelle 7: $\quad$ Berechnung der (Durchschnitts-)Preise

\section{Schritt 8: Umsatzprognosen}

Mit diesen Größen können dann die prognostizierten Jahresumsätze aus den Monats- und den einmaligen Anschlussgebühren insgesamt für das jeweilige Segment und kumuliert über alle Segmente bestimmt werden. Entsprechen die betrachteten Perioden t einem Jahr, sind zur Berechnung des Jahresumsatzes aus Monatsgebühren $U P_{:}^{\text {s.U }}$ die Monatspreise $p_{:}^{\text {s.U }}$ mit $Z$ wölf und dem durchschnittlichen Jahresbestand an Kunden zu multiplizieren. Für die Berechnung des Jahresumsatzes aus einmaligen Anschlussgebühren $U_{G}^{, j, U}$ sind die Anschlussgebühren $\mathrm{g}_{1}^{\mathrm{s} U} \mathrm{U}$ mit dem jährlichen Bestandszuwachs zu multiplizieren.

\begin{tabular}{|l|c|c|}
\hline Bezeichnung & Variable/Formel & Art der Ermittlung \\
\hline $\begin{array}{l}\text { Jahresumsatz aus Monatsgebühren } \\
\text { Jahresumsatz aus einmaligen An- } \\
\text { schlussgebühren }\end{array}$ & $U P_{t}^{s, U}=\frac{m_{t}^{s, U}+m_{t-1}^{s, U}}{2} p_{t}^{s, U} \cdot 12$ & Berechnung \\
\hline $\begin{array}{l}\text { Jahresumsatz je Segment } \\
U G_{t}^{s, U}=\left(m_{t}^{s, U}-m_{t-1}^{s, U}\right) \cdot g_{t}^{s, U}\end{array}$ & Berechnung \\
\hline kumulierter Jahresumsatz & $U_{t}^{s, U}=U P_{t}^{s, U}+U G_{t}^{s, U}$ & Berechnung \\
\hline
\end{tabular}

Tabelle 8: Umsatzprognosen 
Schritt 9: Schätzung der durchschnittlichen Einzahlungsverschiebung

Vor der Überleitung der ermitteiten Umsätze in die Einzahiungen sind die Zahlungsverschiebung $z$ und der Zahlungsausfall $x \in[0 ; 1]$ zu schätzen. ${ }^{33}$

\begin{tabular}{|l|c|c|}
\hline Bezeichnung & Variable/Formel & Art der Ermittlung \\
\hline Tage bis zum Zahlungseingang & $\mathrm{z}$ & Schätzung \\
\hline Zahlungsausfall & $\mathrm{x}$ & Schätzung \\
\hline
\end{tabular}

Tabelle 9: $\quad$ Schätzung der durchschnittlichen Einzahiungsverschiebung und des Zahlungsausfalls

\section{Schritt 10: Berechnung der Einzahlungen}

In einem letzten Schritt wird nun - unter Berücksichtigung der durchschnittlichen Zahlungsverschiebung und der durchschnittlichen Zahlungsausfallrate - aus der Umsatzprognose eine Einzahlungsprognose abgeleitet.

\begin{tabular}{|l|c|c|}
\hline Bezeichnung & Variable/Formel & Art der Ermittlung \\
\hline Einzahlungen & $\mathrm{E}_{\mathrm{t}}^{\mathrm{U}}=\left(\mathrm{U}_{\mathrm{t}-\mathrm{L}}^{\mathrm{U}} \cdot \frac{2}{365}+\mathrm{U}_{\mathrm{t}}^{\mathrm{U}} \cdot \frac{365-z}{365}\right)(1-\mathrm{x})$ & Berechnung \\
\hline
\end{tabular}

Tabelle 10: Berechnung der Einzahlungen

\subsubsection{Die Prognose der Auszahlungen}

Mit dem Ansatz zur Prognose der Auszahlungen wird eine Methodik präsentiert, welche den Übergang von der leistungswirtschaftlichen Betrachtungsweise zur zahlungsbezogenen finanzwirtschaftlichen Sichtweise zuverlässig unterstützt. Folgende Aspekte sind bei der Datenerhebung zu beachten:

- Da bei der Bewertung die Zahlungsgrößen relevant sind, müssen die ermittelten Kosten um die zahlungsunwirksamen Bestandteile (z. B. Abschreibungen) bereinigt werden. So werden beispielsweise die Abschreibungen über die Investitionsauszahlungen berücksichtigt. Für eine Bewertung nach Steuern wären dagegen die Abschreibungen in modifizierter Form anzusetzen.

- Im Allgemeinen sind die Stückkostensätze der variablen Kosten nicht konstant, sondern werden auf Grund beschaffungs- und produktionsseitiger Skaleneffekte von der Absatzmenge determiniert.

- Auch Fixkosten geiten nur innerhalb bestimmter Absatzmengenbereiche als fixx und müssen entsprechend als sprungfix (und damit ebenfalls als von der Absatzmenge abhängig) modelliert werden.

83 Beide Variablen sind hier vereinfachend als konstant über die Zeit angenommen. 


\section{Schritt 1: Identifikation der Wertschöpfungskette mit ihren einzelnen} Wertschöpfungsgliedern

Auf Grund der Komplexität des Getlechtes der zu schätzenden Kosten ist es sinnvoll, im ersten Schritt eine differenziertere Kostenkategorisierung vorzunehmen. Dabei sind die einzelnen Wertschöpfungsglieder entlang der Wertschöpfungskerte ${ }^{34}$ zu identifizieren. Als Wertschöprungsglieder kommen in der vorliegenden Entscheidungssituation beispielsweise die UMTS-Entwicklung und UMTS-Bereitstellung, das Marketing, der Vertrieb, die Auftragsabwicklung und die Kundenbetreuung in Betracht. Für jedes einzelne Wertschöpfungsglied sind die verschiedenen, zu Auszahlungen führenden Komponenten zu identifizieren und - wie nachfolgend beschrieben - zu kategorisieren sowie zu schätzen. ${ }^{85}$

Die zu ermittelnden Auszahlungen können in Auszahlungen, die sich auf in der Bilanz wieder zu findende Positionen beziehen (im Weiteren als Investitionsauszahlungen bezeichnet), und in die Auszahlungen, welche die laufenden auszahlungswirksamen Kosten betreffen, unterschieden werden. Während die Investitionsauszahlungen nunmehr mit der Bezeichnung I und $\mathrm{i}$ abgebildet werden, erfolgt die Darstellung der laufenden auszahlungswirksamen Kosten mit $\mathrm{K}$ und $\mathrm{k}$, wobei die Kleinbuchstaben die Investitionsauszahlungen und die auszahlungswirksamen Kosten pro Stück bezeichnen. Als auszahlungswirksame Kosten und als Investitionsauszahlungen ergeben sich nach Unterteilung in variable (v) und sprungtixe (sf) Größen:

$$
\mathrm{K}=\mathrm{k}_{v}(\mathrm{~m}) \cdot \mathrm{m}+\mathrm{K}_{\mathrm{si}}(\mathrm{m}) \text { und } \mathrm{I}=\mathrm{i}_{\mathrm{v}}(\mathrm{m}) \cdot \mathrm{m}+\mathrm{I}_{\mathrm{sf}}(\mathrm{m}) \cdot{ }^{.56}
$$

Liegt ein relativ hoher Block an zusätzlich zuzuschlüsselnden auszahlungswirksamen Kosten und Investitionsauszahlungen vor, wie es beispielsweise bei der UMTS-Lizenz der Fall ist, so empfiehlt sich eine Unterteilung in direkt ausgelöste auszahlungswirksame Kosten und Investitionsauszahlungen (Index , $\mathrm{d}^{\text {“) }}$ ) sowie in zusätzlich in Anspruch genommene auszahlungswirksame Kosten und Investitionsauszahlungen (Index , in"). Die in Anspruch genommenen auszahlungswirksamen Kosten und Investitionsauszahlungen betreffen jene Auszahlungen. die in Kombination mit anderen Produkten und somit nicht ausschlieBlich durch das betrachtete Produkt - im vorliegenden Beispiel: UMTS - verursacht werden. Somit ist es erforderlich, die entsprechenden Bestandteile verursachungsgerecht aufzuteilen. Schließlich lassen sich folgende entscheidungsrelevante Investitionsauszahlungen und auszahlungswirksame Kosten identifizieren:

$$
I=\left[i_{v}^{d}(m)+i_{v}^{i n}(m)\right] \cdot m+I_{s f}^{d}(m)+I_{s f}^{i n}(m) \text { und } K=\left[k_{v}^{d}(m)+k_{v}^{i n}(m)\right] \cdot m+K_{s f}^{d}(m)+K_{s f}^{i n}(m) .
$$

\section{Schritt 2: Bestimmung der einzeinen Komponenten der auszahlungswirksamen Kosten und der Investitionsauszahlungen}

Im Schritt 2 sind für jedes Wertschöpfungsglied die jeweiligen Komponenten der auszahlungswirksamen Kosten und der Investitionsauszahiungen $k_{v}^{d}(m), \quad k_{v}^{i n}(m), \quad K_{s f}^{d}(m)$, $\mathrm{K}_{\mathrm{sf}}^{\mathrm{n}}(\mathrm{m}), \mathrm{i}_{\mathrm{v}}^{\mathrm{d}}(\mathrm{m}), \mathrm{i}_{\mathrm{v}}^{\mathrm{in}}(\mathrm{m}), \mathrm{I}_{\mathrm{sf}}^{\mathrm{d}}(\mathrm{m})$ und $\mathrm{I}_{s i}^{\text {in }}(\mathrm{m})$ zu ermitteln. Die bei dieser Ermittlung beste-

84 Vgl. zur "neuen“ Mobilfunk-Wertschöpfungskette u. a. BOROWICZ/SCHERM (2002), S. 62 ff.

85 Aus Vereinfachungsgründen werden bei der nachfolgenden formalen Beschreibung die Indizes für das jeweils betrachtete Wertschöpfungsglied und für das betrachtete Jahr $t$ nicht angeführt.

86 Im hier betrachteten Fail handelt es sich bei $i_{v}$ um Investitionen in das kundenbezogene Equipment (Unlaufvermögen). 
henden Probleme der Informationsgewinnung betreffen haupssächlich die Besonderheiten der technischen Produktrealisierung sowie den Entwicklungsstand von Planungsrechnungen und Datenpools in der Unternehmung. Innerhalb dieses Schrittes wird eine möglichst vollständige Identifikation und Schätzung der auszahlungswirksamen Kosten und der Investitionsauszahlungen durch die Analyse entlang der Wertschöprungskette unterstützt.

Als größte Herausforderung kann die Abschätzung des Wertschöpfungsgliedes ,UMTS-Entwicklung und UMTS-Bereitstellung" angesehen werden, weil hierrür - auf Grund der produktspezifischen Besonderheiten und des Innovationsgrades - keine unmittelbaren Vergleichszahlen zur Verfügung stehen und in der Regel auch eine institutionalisierte Kostenrechnung in Anbetracht der technisch komplexen Zusammenhänge keine Hilfestellung leisten kann. Die Ableitung der auszahlungswirksamen Kosten- und die Investitionsauszahlungskomponenten erfolgt vielmehr aus dem technischen Konzept. in dem der Analyst von technischen Spezialisten Informationen in hinreichender Genauigkeit erhalten oder wenigstens nachträglich einfordern soll. Hierbei besteht das Problem, dass zumeist mehrere technische Realisierungsaiternativen möglich sind und die Komponenten sowie Prozesse von der gewählten Realisierung abhängig sind. Da hierüber zum Bewertungszeitpunkt gewöhnlich noch wenig Klarheit besteht, sollte sich im Rahmen der Auszahlungsprognose auf wenige wahrscheinliche Szenarien konzentriert werden. Die entscheidenden Determinanten für die Schätzung der Einkaufspreise sind die technologische Entwicklung, der Wettbewerbsdruck am Einkaufsmarkt und die Vernandlungsposition der bewertenden Unternehmung. Dabei ist zu beachten, dass sich z. B. die Verhandlungsposition nicht nur im Zeitablauf, sondern schon allein durch den Erwerb des Bewertungsobjekts ändern kann. Zur Abschätzung mengenabhängiger auszahlungswirksamer Kosten und Investitionsauszahlungen kann u. a. auf das Erfahrungskurvenkonzept zurückgegriffen werden.

Wie bereits dargestellt, treten auszahlungswirksame Kosten- und Investitionsauszahlungskomponenten mit fixem Charakter - soweit sie sich dem Produkt zurechnen lassen - meist in sprungfixer Form auf, welche dann in Abhängigkeit von der Häufigkeit, vom Ausmaß der Sprünge und vom Vereinfachungsgrad variabel, fix oder sprungfix modelliert werden können. Bei der Auszahlungsprognose ist diesbezüglich zu berücksichtigen, inwieweit Leerkapazitätskosten durch andere Produkte sinnvoll verringert werden können. Voraussetzung hierfür sind funktionierende Schnittstellen zwischen Marketing, Kapazitätsmanagement und Unternehmungsleitung. 


\begin{tabular}{|c|c|c|}
\hline Bezeichnung & Variable/Formel & Art der Ermittlung \\
\hline $\begin{array}{l}\text { auszahlungswirksame Kostenkom- } \\
\text { ponenten }\end{array}$ & $\begin{array}{c}\mathrm{k}_{v}^{\mathrm{d}}(\mathrm{m}), \mathrm{k}_{v}^{\mathrm{nn}}(\mathrm{m}), \mathrm{K}_{j \mathrm{f}}^{\mathrm{d}}(\mathrm{m}), \\
\mathrm{K}_{\mathrm{sf}}^{\mathrm{in}}(\mathrm{m})\end{array}$ & Analyse/Schätzung \\
\hline $\begin{array}{l}\text { Investitionsauszahlungskomponen- } \\
\text { ten }\end{array}$ & $\mathrm{i}_{\mathrm{v}}^{\mathrm{d}}(\mathrm{m}), \mathrm{i}_{\mathrm{v}}^{\mathrm{in}}(\mathrm{m}), \mathrm{I}_{\mathrm{si}}^{\mathrm{d}}(\mathrm{m}), \mathrm{I}_{\mathrm{sf}}^{\mathrm{in}}(\mathrm{m})$ & Analyse/Schätzung \\
\hline auszahlungswirksame Kosten $=$ & $\begin{aligned} K & =\left[k_{v}^{d}(m)+k_{v}^{i n}(m)\right] \cdot m \\
& +K_{s f}^{d}(m)+K_{s f}^{i n}(m)\end{aligned}$ & Berechnung \\
\hline Investitionsauszahlungen $=$ & $\begin{aligned} I & =\left[i_{v}^{d}(m)+i_{v}^{i n}(m)\right] \cdot m \\
& +I_{s f}^{d}(m)+I_{s f}^{i n}(m)\end{aligned}$ & Berechnung \\
\hline
\end{tabular}

Tabelle 11: Bestimmung der Komponenten der auszahlungswirksamen Kosten und Investitionsauszahlungen

Schritt 3: $\quad$ Ermittlung der durchschnittlichen Auszahlungsverschiebung

Analog zum Schritt 9 der Einzahlungsprognose ist hier, z. B. auf Grund eines eingeräumten Zahlungszieles oder auf Grund von Zahlungsverzögerungen, eine durchschnittliche Auszahlungsverschiebung zu berücksichtigen.

\begin{tabular}{|l|c|c|}
\hline Bezeichnung & Variable/Formel & Art der Ermittlung \\
\hline Tage bis zum Zahlungsausgang & $y$ & $\begin{array}{c}\text { Unternehmungs- } \\
\text { entscheidung/ } \\
\text { Schätzung }\end{array}$ \\
\hline
\end{tabular}

Tabelle 12: $\quad$ Ermittlung der durchschnittlichen Auszahlungsverschiebung

\section{Schritt 4: Berechnung der Auszahlungen}

Die Addition der in Schritt 2 berechneten Werte für alle Wertschöpfungsglieder ergibt - gegebenenfalls unter erforderlicher Berücksichtigung der durchschnittlichen Auszahlungsverschiebung - die periodenbezogene Gesamtauszahlungsprognose. ${ }^{37}$

\begin{tabular}{|l|c|c|}
\hline Bezeichnung & Variable/Formel & Art der Ermittlung \\
\hline $\begin{array}{l}\text { Addition der Auszahlungskompo- } \\
\text { nenten }=\end{array}$ & $\mathrm{A}_{t}^{\mathrm{wK}}=\mathrm{K}^{\mathrm{wK}}+I^{\mathrm{wK}}$ & Berechnung \\
\hline $\begin{array}{l}\text { Kumulation über die Wertschöp- } \\
\text { fungskette }=\end{array}$ & $\mathrm{A}_{\mathrm{t}}^{U}=\sum_{\mathrm{wk}} \mathrm{A}_{t}^{\mathrm{wk}}$ & Berechnung \\
\hline Auszahlungen $=$ & $\mathrm{A}_{t}=\mathrm{A}_{\mathrm{t}-1}^{\mathrm{U}} \cdot \frac{\mathrm{y}}{365}+\mathrm{A}_{t}^{\mathrm{U}} \cdot \frac{365-\mathrm{y}}{365}$ & Berechnung \\
\hline
\end{tabular}

Tabelle 13: Berechnung der Auszahlungen

37 An dieser Stelle wird der Index t wieder eingeblendet. 
Der dargestellte heuristische Ansatz bietet eine Hilfesteilung zur Enmittung der reievanten Effolge im Sinne des bereits dargestellten Zusammenhangs: $Z \ddot{U}_{i}=E_{t}^{m B O}-E_{i}^{u B O}-A_{t}^{m B O}-A_{t}^{o B O}$.

\section{Die Transformation der Zukunftserfolge}

\subsection{Das Zukunftserfolgswertverfahren}

Die unter Berücksichtigung der grundsätzlichen Prinzipien zur Zukunftserfolgsabgrenzung und u. U. mithilfe des dargestellten heuristischen Ansatzes ermittelten Informationen über Streuungen, Bandbreiten und Interdependenzen der künftigen Zahlungsüberschüsse bilden den Ausgangspunkt zur Transformation dieser Informationen in einen Wert, welcher als Entscheidungsgrundlage dienen soll.

Um der Notwendigkeit einer Reduktion der mit der Zukunftserfolgsermittlung ,erzielten" Komlexität des Bewertungskalküls zu entsprechen, werden nachfolgend die Möglichkeiten und Grenzen des Verfahrens des Zukunftserfolgswertes, eines Partialmodells, betrachtet. Im Partialmodell fungieren als Rechengröße nicht die Ausschüttungen an die Unternehmungseigner, sondern einzelne Zahiungsreihen. Das Dualitätstheorem der linearen Optimierung ${ }^{38}$ ermöglicht es, dass durch das Bewertungsobjekt induzierte Zanlungsströme von der bewertenden Unternehmung dezentral beurteilt werden können und gleichzeitig die getroffene Zielsetzung verfolgt wird. Bei Verwendung der investitionstheoretisch korrekten Steuerungszinsfüße in Partialmodellen bleiben die Interessen der Eigner gewahrt. ${ }^{99}$

Während der Terminus des Entscheidungswertes den Zweck des Bewertungskalküls hervorhebt, stellt der Zukunftserfolgswert begrifflich auf die spezielle Wertermittlungsmethode ab..$^{90}$ Im Unterschied zum Totalmodeli $1^{91}$, in dem der Nutzen des Basisprogramms mit dem des Bewertungsprogramms verglichen wird, erfolgt beim Zukunftserfolgswertverfahren eine Gegenüberstellung des Bewertungsobjekts mit dem vorteilhaftesten Alternativinvestitionsprogramm. Die Ermittlung des Entscheidungswertes mit dem Zukunftserfolgswertverfahren hat dabei mit dem Prinzip der Subjektivität, dem Prinzip der Gesamtbewertung und dem Prinzip der Zukunftsbezogenheit die drei fundamentalen Grundsätze der Bewertung zu berücksichtigen.

Der Zukunfiserfolgswert ZEW als Variante des Gegenwartswertkalküls entspricht dem Barwert der mit den Kalkulationszinsfußen ${ }^{92} r_{t}$ abgezinsten künftigen Erfolge $Z Z_{t}$ des Bewer-

88 Siehe zur Dualitätstheorie u. a. DANTZIG (1966), S. 148 ff., HERING (1995), S. 78 ff.

89 Vgl. HERING (1999), S. 19 und zum Beweis für den vorliegenden Zusammenhang HERING (1995), S. 90.

90 Vgl. WATSCHKE (1975), S. $23 \mathrm{f}$.

$9 \mathrm{~S}$ Siehe hierzu z. B. die Bewertung mit dem Zusiands-Grenzpreismodell (ZGPM) in HERNG (1999), S. 29 ff. und S. 188 f., HERING (2000), S. 437 ff; siehe zur Erweiterung des Modells um Produktionsaspekte HERING (2002), S. 74 ff. und um nichtlineare Aspekte PFAFF/PFEIFFER/GATHGE (2002); zur Anwendung des ZGPM siehe u. a. BROSEL (2002), S. 91 ff., OLARCH (2002), S. 686 ff., BROSEL (2003), MATSCHKE/BROSEL (2003).

92 Zur Bedeutung des Kalkulationszinsfußes in der Untemehmungsbewertung vgl. z. B. B.tLLIWESER (2002), S. 736. 
tungsobjekts im Sinne von Einzahlungsüberschüssen. Die Kalkulationszinsfüße dienen als Vergleichsmaßstab und resultieren aus der besten alternativen Kapitalverwendungsmöglichkeit des Entscheidungssubjekts. Unter Vernachlässigung nichtfinanzieller Restriktionen ${ }^{93}$ ergibt sich der Zukunftserfolgswert von Telekommunikationsunternehmungen oder von ihren abgrenzbaren Untemehmungsbestandteilen gemäß folgender Formel der so genannten „vereinfachten" Bewertung::44

$$
\mathrm{ZEW}=\sum_{\mathrm{i}} \frac{\mathrm{Z \textrm {U } _ { 1 }}}{\prod_{\tau=1}^{t}\left(1+\mathrm{r}_{\tau}\right)}
$$

Die Kenntnis der Grenzzinsfüße einer jeden Periode ist also die Grundlage der dezentralen Anwendung des Partialmodells „Zukunftserfolgswertverfahren“. Die Ermittlung der für die einzelnen Perioden gültigen endogenen Grenzzinsfüße erfordert auf dem unvollkommenen Kapitalmarkt allerdings eine Totalbetrachtung. ${ }^{95} \mathrm{Da}$ die Steuerungszinsfüße erst durch die optimale Lösung des Totalmodells selbst definiert werden, wird vom Dilemma der wertmäßigen Kosten oder der Lenkpreistheorie ${ }^{36}$ gesprochen. Die hinsichtlich des Kalkulationszinses verbreitete Forderung nach dem internen Zins des günstigsten Vergleichsobjekts ${ }^{77}$ ist lediglich für den Spezialfall einperiodiger Grenzobjekte zu vertreten. ${ }^{98}$ Die Kalkulationszinsfüße werden auf dem unvollkommenen Kapitalmarkt durch das Entscheidungsfeld und durch die individuellen Konsumpräferenzen des jeweiligen Bewertungssubjekts determiniert. ${ }^{99}$

In der praktischen Anwendung ist jedoch zu erwarten, dass diese Lenkpreisproblematik durch das hauptsächlich mit der Zukunftserfolgsermittlung auftretende Unsicherheitsproblem stark überlagert wird. Deshalb ist anzuraten, sich bei der Bewertung nicht auf einen bestimmten Prozentsatz festzulegen, sondern eine Bandbreite von potentiellen Grenzzinsfüßen zu berücksichtigen. ${ }^{100}$ Ist sich die bewertende Unternehmung über die Schwächen des Zukunftserfolgsmodells bewusst und berïcksichtigt sie diese bei der Anwendung des zur Verfügung stehenden investitionstheoretisch fundierten „Bewertungsinstrumentariums", ermöglicht dieses Partialmodell eine zuverlässige Wertermittlung für Akquisitionsobjekte der „Alten“ und der „Neuen Wirtschaft“ sowie eine theoretisch fundierte Entscheidungsunterstützung. ${ }^{101}$

93 Zur Problematik der Benicksichtigung nichtfinanzieiler Restriktionen im Zukunftserfolgswertverfahren siehe BRÖSEL (2002), S. $157 \mathrm{ff}$.

94 Vgi. hierzu die Austührungen zur ,vereinfachten“ Bewertung sowie die Formel (21) in LAUX/FRANKE (1969), S. $210 \mathrm{ff}$.

95 Gemäß dem Marginalprinzip sind die Grenzobjekte des besten altemativen Portefeuilles ais Vergleichsobjekte heranzuziehen; vgl. hierzu MOXTER (1983), S. 141 sowie austührlich HERNG (1999), S. 34 ff. Zur Wahl des Kalkulationszinses in der Praxis der Unternehmungsbewertung siche die empirische Analyse in PRETZE/WALKER (1995), S. $205 \mathrm{ff}$.

96 Siehe HIRSHLEIFER (1958), S. 340, HAX (1964), HERING (1995), S. 69 ff.. ROLLBERG (2001), S. 136 ff.

97 Vgl. beispielsweise MÜNSTERMANN (1966), S. 151.

98 Siehe hierzu HERNG (1999), S. 38 f.

99 Vgl. HERING (1995), S. 12.

100 Siehe zu den Problemen bei der Wahl des Kalkulationszinsfüßes BALLWIESER (2002).

101 Vgl. OLBRICH (2002), S. 688. Zur ausführlichen Kritik an den für die „Neue Wirtschaft“ vorgeschlagenen Bewertungsmethoden, der Bewertung mithilfe der Geldverbrennungsrate, dem Muitiplikatorverfahren und dem Sachoptionsvertahren, siehe OLBRICH (2002), S. $688 \mathrm{ff}$. 


\subsection{Die Berücksichtigung der Unsicherheit}

Die Erfolgsschätzungen in der Telekommunikationsbranche sind in Anbetracht der großen Unsicherheit durch mehrwertige Erwartungen geprägt. Grundlage der Bewertung bilden die zur Verfügung gestellten Erfolgserwartungen, die (schon unter Anwendung subjektiver Komplexitätsreduktion ${ }^{102}$ ) auf subjektive Bandbreiten eingeengt und denen bestenfalls durch fundierte Schätzungen ermittelte Eintrittswahrscheinlichkeiten zugeordnet worden sind. Unter diesen Bedingungen ist eine ,optimale“ Lösung des zielsetzungsdefekten ${ }^{103}$ Bewertungsproblems ex ante nicht definiert. Heuristische Verfahren ${ }^{104}$ zur Berücksichtigung der Mehrwertigkeit der Zukunftserwartungen des Bewertungssubjekts lassen sich in Unsicherheit verdichtende und Unsicherheit offen legende Bewertungsmethoden unterteilen.

Bei Unsicherheit verdichtenden Bewertungsmethoden wird die Unsicherheit entweder auf der Ebene der Eingangsdaten oder auf der Ebene des Zielwertes komprimiert. Die Berücksichtigung des Unsicherheitsproblems auf der Ebene der Eingangsdaten kann einerseits durch die Verwendung von mit Sicherheitszu- oder -abschlägen korrigierten Planungsdaten oder andererseits durch die Einengung der für unsicher gehaltenen Parameter auf faktische Einwertigkeit erfolgen. Diese „Berichtigung“" der Zahlungsgrößen ${ }^{105}$ oder der Zinssätze ${ }^{106}$ ermöglicht anschließend eine Bewertung mit deterministischen Modellen. ${ }^{107}$ Der Nachteil dieser Methoden besteht jedoch darin, dass Planungsdaten willkürlich korrigiert, Parameterstreuungen nicht berücksichtigt sowie die Dynamik der Zustände im Zeitablauf nicht abgebildet werden und somit die Aussagefähigkeit des ermirtelten (Punkt-)Wertes gering ist. ${ }^{108}$ Die Komprimierung der Unsicherheit auf der Ebene des Zielwertes erfolgt hingegen dergestalt, dass die Informationen über die Bandbreiten und die Verteilung der mehrwertigen Eingangsgrößen des Bewertungsproblems ausdrücklich dazu genutzt werden, um hieraus einen einheitlichen

102 Bezüglich der im Vorfeld vollzogenen Komplexitätsreduktion sei insbesondere auf die diskontinuierliche Betrachtung eventuell kontinuierlich anfallender Zahlungsströme hingewiesen.

103 Zu Strukturdefekten bei Entscheidungsproblemen siehe ADAM(1996), S. 10 ff.

104 Siehe zu nachfolgenden Ausführungen zur Berücksichtigung der Unsicherheir die ausführliche Analyse von Planungsmethoden unter Unsicherneit in HERING (1995), S. 173 ff. sowie die zusamumentassenden Darstellungen in ROLLBERG (1999), S. $106 \mathrm{ff}$. und ROLLBERG (2001), S. 189 ff.

105 Die Anpassung der Zahlungsgrößen findet z. B. im Rahmen der Sicherheitsäquivalenzmethode statt. Bei dieser Methode erfolgt eine Aggregation der in Bandbreiten oder als subjektive Wahrscheinlichkeitsverteilungen vorliegenden Zahiungsgrößen in so genannte Sicherneitsäquivalente. Auf der Basis des BERvorLLI-Prinzips und mit der erforderlichen Kenntnis der Risikopräferenzen des Entscheidungssubjekts werden die unsicheren Zukun ttserfolgsströme in einen sicheren Strom ransformiert, den das Bewerungssubjekt als gleichwertig einschätzt. Mit anderen Worten. unter dem sicherheitsäquivalenten Erfolg wird derjenige sichere Erfolg verstanden, der dem Bewertungssubjekt genauso viel wert ist, wie die geschätzte unsichere Erfolgsbandbreite. Soll die Entscheidungswertfindung nicht zu einem intuitiven Abwägungsprozess führen, sind - was enorme praktische Schwierigkeiten erwarten lässt - bei der Bestimmung der Sicherheitsäquivaiente die Risikonutzenfunktionen aller Bewertungssubjekte zu berïcksichtigen; siehe BALLWIESER (1981), S. $101 \mathrm{ff}$.

106 Die Anpassung der Zinssätze erfolgt beispielsweise bei der Risikozuschlagsmethode. Hierbei werden zur Entscheidungswertermittlung die Erwartungswerte der Einzahlungsüberschüsse und willkürlich risikoangepasste Zinssätze verwendet. Die Höhe des gewähiten Zu- oder Abschiags zur Berücksichtigung des Risikos ist nicht rational begründbar. Vgl. zur Risikozuschlagsmethode und zur Kritik HERING (1995), S. 183 ff.

107 Beide Vorgehensweisen werden jedoch auch aus der Sicht der Wirtschaftsprüfer vorgeschlagen; vgl. INSTITUT DER WIRTSCHAFTSPRUFER (2000), S. 333.

108 Siehe HERNG (1995), S. 178. 
Punktwert als Handlungsempfehlung zu bestimmen. ${ }^{109}$ Bei allen vorgestellten Varianten der Unsicherheit verdichtenden Methoden wird unter Informationsverlust versucht, die mehrwertigen Erwartungen des komplexen Bewertungsproblems in einem Punktwert zu komprimieren, um somit die Unsicherheit künstlich „wegzurechnen“. Dem Bewertungssubjekt wird schließlich ein einwertiger Entscheidungswert mit eher geringer Aussagekraft geliefert. ${ }^{110}$

Als Ergebnis der Unsicherheit offen legenden (oder aufdeckenden) Bewertungsmethoden wird dem Entscheidungssubjekt der Entscheidungswert ais Bandbreite oder als Verteilung zur Verfügung gestellt. Da bei Unsicherheit eine „optimale“ Lösung des Bewertungsproblems ex ante nicht definiert ist, entsprechen Bandbreiten viel mehr der Natur des Entscheidungswertes. Zur Aufdeckung der Auswirkungen der Unsicherheit kann auf die Sensitivitäts- ${ }^{i 11}$ und die Risikoanalyse ${ }^{12}$ zurückgegriffen werden. ${ }^{1 / 3}$

Die Transformation der durch Fachleute der Telekommunikationsbranche geschätzten Erfolgserwartungen ender im Rahmen der Entscheidungsfunktion mit der Ermittlung eines Entscheidungswertes. Dieser Wert soll dem Entscheidungssubjekt zur Entscheidungsunterstützung dienen. Die Abwägung zwischen Preis und Entscheidungswert, in welche die individuellen Risikoneigungen des Entscheidungssubjekts einfließen, schließt sich an den Bewertungsvorgang an und erfordert transparente Informationsgrundlagen. ${ }^{14}$ Auf Grund der mangelnden Zweckmäßigkeit von Unsicherheit verdichtenden Methoden liegt die Verwendung von Unsicherheit offen legenden Bewertungsmethoden nahe. Diese Methoden schaffen die notwendige „Transparenz hinsichtlich der subjektiv für möglich gehaltenen Entscheidungskonsequenzen [...] [und dienen somit] in anschaulicher und nachvollziehbarer Form"1/15 als Entscheidungsgrundlage.

109 Unter diese Methoden fallen beispieisweise die stochastische Optimierung und die unscharfe lineare Optimierung. Die stochastische Optimierung interpretiert die eirzelnen Eingangsdaten des Bewertungsproblems als Zufallsvariable mit bekannten Wahrscheinlichikeitsvertelungen, um letztendlich die mehrwertigen Erwartungen zu einem Purktwert zu verdichten; vgl. ROLLBERG (1999), S. 107. Im Unterschied dazu erfolgt die Komprimierung der Unsicherheit im Rahmen der auf die Fuzzy Lagic zuruickzuführenden unscharfen linearen Optimierung lediglich unter Berücksichtigung der Bandbreiten der einzelnen Koeffizienten des Bewertungsproblerns; vgl. HERING (1995), S. 218 ff., KEUPER (1999), S. 93 ff.

110 Für MOXTER sind einwertige Erfolgsprognosen nicht realitätsgerecht, eine Komprimierung der Daten auf einen Punktwert als Entscheidungswert hält MOXTER indessen für stathaft: siehe MOATER (1983), S. 117 und S. 156.

111 Mit Sensitivitätsanalysent wird die Emprindlichkeit der Bewertungsergebnisse auf die Veränderung der Planungsdaten untersucht; vgl. weiterführend z. B. OINKELBACH (1979).

12 Die simulative und die analytische Methode der Risikoanalyse leiten aus den gegebenen Verteilungen der Planungsgrößen eine statistische Verteilung für den Zieiwert ab; vgl. weitertührend u. a. HERTZ (I964), HERNG (1995), S. 207 ff.

113 Siehe zur Befürwortung der Unsicherheit offenlegenden Bewertungsmethoden beispielsweise COENENBERG (1970), S. 804, SIECEL (1992), S. 26, HERING (1999), S. 26 f.

114 Siehe auch HERING (1999), S. 26.

115 HERNG (1995), S. 181 (Hervorhebungen im Originai). 


\section{$5 \quad$ Eine Zusammenfassung}

In der theoriegeleiteten Unternehmungsbewertungsdiskussion wird bezüglich des ohne Zweifel gravierenden Datengewinnungsproblems auf die Einbeziehung von Branchenexperten verwiesen. Vor diesem Hintergrund stellt dieser Beitrag mit den Erläuterungen za den Bewertungsanlässen, den Konzeptionen, den Funktionen und den Grundsätzen funktionaier Bewertungstheorie die Grundlagen für die Bewertung von Telekommunikationsunternehmungen und von deren Unternehmungsteilen bereit. Im Mittelpunkt stehen anschließend die Datengewinnung sowie die Transformation der zukünftigen Erfolge in einen Entscheidungswert. Die Abgrenzung und Quantifizierung der relevanten Zukunftserfolge wird mittels einer entwickelten Heuristik unterstützt. Darin werden ein Erlösmodell sowie ein Investitions- und Kostenmodell integriert. Diese liefern mit der transparenten Offenlegung der Parameter, Variablen und letztlich Treiber die Basis für eine adäquate unsicherheitsberücksichtigende Transformation der Zukunftserfolge in die gesuchte Größe. Der Ansatz lässt sich beliebig erweitern, wie z. B. um die Modellierung einer durchschnittlichen Kundenverweildauer oder um die Einbeziehung in vollständige Finanzpläne, und wurde auch bereits für andere Anwendungsfelder, wie Bewerting von Markterschließungsstrategien im Festnetzgeschäft und für die Bewertung von Geschäftsmodellen für ,Application Service Providing " erfolgreich eingesetzt.

\section{Symbolverzeichnis}
$A_{\mathfrak{i}}^{\mathrm{mBO}}$
Auszahlungen der bewertenden Unternehmung mit dem Bewertungsobjekt in $\mathrm{t}$
$A_{t}^{\mathrm{OBO}}$
$\alpha_{t}^{\text {s.U }}$
$\beta_{h . t}^{\text {s.U }}$
Auszahlungen der bewertenden Unternehmung ohne dem Bewertungsobjekt in $\mathrm{t}$
$\mathrm{E}_{\mathrm{t}} \quad$ Einzahlungen in $\mathrm{t}$
$E_{t}^{m B O}$
unternebmungsbezogener segmentspezifischer Potenzialausschöpfungskoef- fizient
$E_{i}^{\square B O}$
Einzahlungen der bewertenden Unternehmung mit dem Bewertungsobjekt in $t$
$\mathrm{g}_{h, \mathrm{~L}}^{\mathrm{s} M}$
Einzahlungen der bewertenden Unternehmung ohne dem Bewertungsobjekt in $\mathrm{t}$
marktbezogener segmentspezifischer Absatzpreis pro Produktvariante (ein- malige Anschlussgebühr) 


\begin{tabular}{|c|c|}
\hline$g_{h, t}^{s, U}$ & $\begin{array}{l}\text { unternehmungsbezogene segmentspezifische Anschlussgebühr pro Produkt- } \\
\text { variante }\end{array}$ \\
\hline $\mathrm{g}_{\mathrm{i}}^{\mathrm{s}, U}$ & $\begin{array}{l}\text { unternehmungsbezogene segmentspezifische durchschnittliche Anschluss- } \\
\text { gebühr }\end{array}$ \\
\hline $\mathrm{b}$ & Index für Produktvarianten am Markt \\
\hline I & Investitionsauszahlung \\
\hline$I_{s f}^{d}(m)$ & direkt ausgelöste sprungfixe Investitionsauszahlungen \\
\hline $\mathrm{i}_{v}^{\mathrm{d}}(\mathrm{m})$ & direkt ausgelöste variable Investitionsauszahlungen \\
\hline $\mathrm{I}_{\mathrm{sf}}^{\mathrm{in}}(\mathrm{m})$ & in Anspruch genommene sprungfixe Investitionsauszahlungen \\
\hline$i_{v}^{\text {in }}(m)$ & in Anspruch genommene variable Investitionsauszahlungen \\
\hline $\mathrm{K}$ & auszahlungswirksame Kosten \\
\hline $\mathrm{K}_{\mathrm{sf}}^{\mathrm{d}}(\mathrm{m})$ & direkt ausgelöste auszahlungswirksame sprungfixe Kosten \\
\hline $\mathrm{k}_{\mathrm{v}}^{\mathrm{d}}(\mathrm{m})$ & direkt ausgelöste auszahlungswirksame variable Stückkosten \\
\hline $\mathrm{K}_{\mathrm{si}}^{\mathrm{in}}(\mathrm{m})$ & in Anspruch genommene auszahlungswirksame sprungfixe Kosten \\
\hline $\mathrm{k}_{\mathrm{v}}^{\mathrm{in}}(\mathrm{m})$ & in Anspruch genommene auszahlungswirksame variable Stückkosten \\
\hline M & Index für den Marktbezug \\
\hline m bzw. $m_{t}^{s, u}$ & Kundenzahl/Verkaufsmenge \\
\hline $\mathrm{MA}_{\mathrm{t}}^{\mathrm{s}, \mathrm{U}}$ & unternehmungsbezogener segmentspezifischer Marktanteil \\
\hline $\mathrm{P}_{\mathrm{h}, \mathrm{t}}^{\mathrm{s}, \mathrm{M}}$ & $\begin{array}{l}\text { marktbezogener segmentspezifischer Absatzpreis pro Produktvariante (Mo- } \\
\text { natspreise) }\end{array}$ \\
\hline $\mathrm{p}_{\mathrm{h}, \mathrm{i}}^{\mathrm{s.U}}$ & $\begin{array}{l}\text { unternehmungsbezogener segmentspezifischer Monatspreis pro Produktvari- } \\
\text { ante }\end{array}$ \\
\hline $\mathrm{p}_{\mathrm{t}}^{\mathrm{s}, \mathrm{U}}$ & $\begin{array}{l}\text { unternehmungsbezogener segmentspezifischer durchschnittlicher Monats- } \\
\text { preis }\end{array}$ \\
\hline $\mathrm{PP}_{\mathrm{t}}^{\mathrm{s}}$ & Penetrationsrate des Kundensegments s in der Periode $t$ \\
\hline$q_{h, t}^{s, u}$ & $\begin{array}{l}\text { unternehmungsbezogener segmentspezifischer Preisanpassungskoeffizient } \\
\text { pro Produktvariante }\end{array}$ \\
\hline$r_{t}$ & ndogener Grenzzinsfü $\beta$ der Periode $\iota$ \\
\hline
\end{tabular}


s

$S^{5}$

t

U

$U^{s, U}$

$U_{t}^{u}$

$\mathrm{UG}_{i}^{\mathrm{s.}} \mathrm{U}$

$\mathrm{UP}_{\mathrm{t}}^{\mathrm{s}, \mathrm{US}}$

WK

$\mathrm{x}$

y

z

ZEW

$\mathrm{ZU}:$

$\mathrm{ZÜ}{ }_{\mathrm{t}}^{\mathrm{mBO}}$

$\mathrm{ZU} \ddot{\imath}^{\mathrm{oBO}}$
Index für Kundensegmente

Umfang des Kundensegments $s$ in der Periode $t$

Index für den Zeitpunkt; Periode als Zeitraum zwischen den Zeitpunkten $\mathrm{t}-1$ und $\mathrm{t}$

Index für den Untemehmungsbezug

unternehmungsbezogener Jahresumsatz je Segment

unternehmungsbezogener kumulierter Jahresumsatz

unternehmungsbezogener Jahresumsatz aus einmaligen Anschlussgebühren je Segment

unternehmungsbezogener Jahresumsatz aus Monatsgebühren je Segment

Glieder der Wertschöpfungskette der Unternehmung

Zahlungsausfall

Tage bis zum Zahlungsausgang

Tage bis zum Zahlungseingang

Zukunftserfolgswert

bewertungsrelevanter Vorteilsstrom im Sinne der Zahlungsüberschüsse in t

Zahlungsüberschuss der bewertenden Untemehmung mit dem Bewertungsobjekt in $t$

Zahlungsüberschuss der bewertenden Unternehmung ohne dem Bewertungsobjekt in $\mathrm{t}$ 


\section{Quellenverzeichnis}

ADAM, D. (1996): Planung und Entscheidung, 4. Aufl., Wiesbaden 1996.

ALLER, W. (1926/1927): Die Bewertung der Unternehmung als Wirtschaftseinheit, in: Welt des Kaufmanns, 8. Jg. (1926/1927), S. 41-46.

BALLWESER, W. (1981): Die Wahl des Kalkulationszinsfußes bei der Unternehmensbewertung unter Berücksichtigung von Risiko und Geldentwertung, in: BFuP, 33. Jg. (1981), S. 97-114.

BALLWIESER, W. (2002): Der KalkulationszinsfuB in der Untemehmensbewertung: Komponenten und Ermittlungsprobleme, in: WPg, 55. Jg. (2002), S. 736-743.

BALLWIESER, W. / LEUTHIER, R. (1986): Betriebswirtschaftliche Steuerberatung: Grundprinzipien, Verfahren und Probleme der Untemehmensbewertung, in: DStR, 24. Jg. (1986), S. $545-$ 551 und S. 604-610.

BÖRNER, D. (1980): Unternehmensbewertung, in: ALBERS, W. ET AL. (Hrsg.), Handwörterbuch der Wirtschaftswissenschaften, Bd. 8, Stuttgart et al. 1980, S. 111-123.

BOROWICZ, F. / SCHERM, E. (2002): Wettbewerb im Mobilfunkmarkt - die Folgen von UMTS, in: KEUPER, F. (Hrsg.), Electronic Business und Mobile Business, Wiesbaden 2002, S. 677708.

BRÖSEL, G. (2002): Medienrechtsbewertung. Der Wert audiovisueller Medienrechte im dualen Rundfunksystem, Wiesbaden 2002.

BRÖSEL, G. (2003): Zur Bewertung von Film- und Übertragungsrechten aus Sicht öffentlichrechtlicher Fernsehveranstalter, erscheint in: ZögU, 26. Jg. (2003), Heft 1.

BUSSE VON COLBE, W. (1957): Der Zukunftserfolg, Wiesbaden 1957.

COENENBERG, A. G. (1970): Unternehmungsbewertung mit Hilfe der Monte-Carlo-Simulation, in: ZfB, 40. Jg. (1970), S. 793-804.

COENENBERG, A. G. / SIEBEN, G. (1976): Unternehmungsbewertung, in: GROCHLA, E. / WITTMANN, W. (Hrsg.), Handwörterbuch der Betriebswirtschaft, 4. Aufl., Stuttgart 1976, Sp. 40624079.

DANTZIG, G. B. (1966): Lineare Programmierung und Erweiterungen, Berlin, Heidelberg, New York 1966.

DECHANT, H. / BRASSLER, A. (2003): Business-Case: Internet, in: BRÖSEL, G. / KEUPER, F. (Hrsg.), Medienmanagement, München/Wien 2003, S. 65-84.

DECHANT, H. / TROST, R. (2001): Wirtschaftlichkeitsbewertung von Produktinnovationen im Telekommunikationssektor, in: Joumal für Betriebswirtschaft, 51. Jg. (2001), S. 234-242.

DINKELBACH, W. (1979): Sensitivitätsanalysen, in: BECKMANN, J./MENGES, G. / SELTEN, R. (Hrsg.), Handwörterbuch der mathematischen Wirtschaftswissenschaften, Bd. 3, Wiesbaden 1979 , S. 243-247.

ENGELS, W. (1962): Betriebswirtschaftliche Bewertungslehre im Licht der Entscheidungstheorie, Köln/Opladen 1962. 
GOETZKE, W. SIEBEN, G. (Hrsg.) (1977): Moderne Unternehmungsbewertung und Grundsätze ihrer ordnungsmäßigen Durchführung, Köln 1977.

GOSSE.V, H. H. (1854): Entwickelung der Gesetze des menschlichen Verkehrs, und der daraus fließenden Regeln für menschliches Handeln, Braunschweig 1854.

$H A X, H$. (1964): Investitions- und Finanzplanung mit Hilfe der linearen Programmierung, in: $\mathrm{ZfbF}, 16$. Jg. (1964), S. 430-446.

HERING, TH. (1995): Investitionstheorie aus der Sicht des Zinses, Wiesbaden 1995.

HERING, TH. (1999): Finanzwirtschaftliche Untemehmensbewertung, Wiesbaden 1999.

HERING. TH. (2000): Konzeptionen der Unternehmensbewertung und ihre Eignung für mittelständische Unternehmen, in: BFuP, 52. Jg. (2000), S. 433-453.

HERING, TH. (2002): Bewertung von Produktionsfaktoren, in: KEUPER, F. (Hrsg.), Produktion und Controlling, Festschrift für M. Layer, Wiesbaden 2002, S. 57-81.

HERING, TH. / OLBRICH, M. (2001): Zur Bewertung von Mehrstimmrechten, in: ZfbF, 53. Jg. (2001), S. 20-38.

HERTZ, D. B. (1964): Risk Analysis in Capital Investment, in: Harvard Business Review, 42. Jg. (1964), S. 95-106.

HIRSHLEIFER, $J$. (1958): On the Theory of Optimal Investment Decision, in: Joumal of Political Economy, 66. Jg. (1958), S. 329-352.

HOMMEL, W. / BRAUN, I. / SCHMOTZ, TH. (200L): Neue Wege in der Untemehmensbewertung?, in: DB, 54. Jg. (2001), S. 341-347.

INSTITUT DER WIRTSCHAFTSPRUFER (1983): Stellungnahme HFA 2/1983: Grundsätze zur Durchfuhrung von Unternehmensbewertungen, in: WPg, 36. Jg. (1983), S. 468-480.

INSTITUT DER WIRTSCHAFTSPROFER (2000): IDW Standard: Grundsätze zur Durchführung von Unternehmensbewertungen (IDW S 1), in: WPg, 53. Jg. (2000), S. 825-842.

KAFER, K. (1996): Zur Bewertung der Unternehmung, Nachdruck von Aufsätzen Karl Käfers aus den Jahren 1946 bis 1973 zum 98. Geburtstag des Autors, hrsg. von C. HELBLING, Zürich 1996 .

KEUPER, F. (1999): Fuzzy-PPS-Systeme - Einsatzmöglichkeiten und Erfolgspotentiale der Theorie unscharfer Mengen, Wiesbaden 1999.

KEUPER, F. (2002): Unscharfe, kapitalwertbasierte Verfahren zur Unternehmensbewertung, in: ZfB, 72. Jg. (2002), S. 457-476.

KOPPELMANN, U. (1997): Produktmarketing, 5. Aufl., Berlin 1997.

KOTLER, P. / BLIEMEL, F. (2001): Marketing Management, 10. Aufl., Stuttgart 2001.

LACKMANN, F. (1962): Theorien und Verfahren der Unternehmungsbewertung, 2. Aufl., Berlin 1962.

LAUX, H. / FRANKE, G. (1969): Zum Problem der Bewertung von Untemehmungen und anderen Investitionsgütern, in: Unternehmensforschung, 13. Jg. (1969), S. 205-223.

LUTZ. H. (1981): Zum Konsens und Dissens in der Unternehmensbewertung, in: BFuP, 33. Jg. (1981), S. 146-155. 
MANDL, G. / RABEL, K. (1997): Unternehmensbewertung, Wien, Frankfurt am Main 1997.

MATSCHKE, M. J. (1969): Der Kompromiß als betriebswirtschaftliches Problem bei der Preisfestsetzung eines Gutachters im Rahmen der Untemehmungsbewertung, in: ZfbF, 21. Jg. (1969), S. 57-77.

MATSCHKE, M. J. (1971): Der Arbitrium- oder Schiedsspruchwert der Unternehmung - zur Vermittlerfunktion eines unparteiischen Gutachters bei der Unternehmungsbewertung -, in: BFuP, 23. Jg. (1971), S. 508-520.

MATSCHKE. M. J. (1972): Der Gesamtwert der Unternehmung als Entscheidungswert, in: BFuP, 24. Jg. (1972), S. 146-161.

MATSCHKE, M. J. (1975): Der Entscheidungswert der Unternehmung, Wiesbaden 1975.

MATSCHKE, M. J. (1976): Der Argumentationswert der Unternehmung - Unternehmungsbewertung als Instrument der Beeinflussung in der Verhandlung, in: BFuP, 28. Jg. (1976), S. 517-524.

MATSCHKE, M.J. (1979): Funktionale Untemehmungsbewertung, Bd. II, Der Arbitriumwert der Unternehmung, Wiesbaden 1979.

MATSCHKE, M.J. (1981): Unternehmungsbewertung in dominierten Konfliktsituationen am Beispiel der Bestimmung der angemessenen Barabfindung für den ausgeschlossenen oder ausscheidungsberechtigten Minderheits-Kapitalgesellschafter, in: BFuP, 33. Jg. (1981), S. 115129.

MATSCHKE, M. J. (1984): Die Bewertung ertragsschwacher Unternebmungen bei der Fusion, in: BFuP, 36. Jg. (1984), S. 544-565.

MATSCHKE, M. J. (1990): Substanzwert in der Unternehmensbewertung, in: LÜCK, W. (Hrsg.), Lexikon der Betriebswirtschaft, Berlin 1990, S. 1106-1109.

MATSCHKE, M. J. (1993): Einige grundsätzliche Bemerkungen zur Ermittlung mehrdimensionaler Entscheidungswerte der Unternehmung, in: BFuP, 45. Jg. (1993), S. 1-24.

MATSCHKE, M. J. (1995): Unternehmungsbewertung: Anlässe und Konzeptionen, in: CORSTEN, H. (Hrsg.), Lexikon der Betriebswirtschaftslehre, 3. Aufl., München, Wien 1995, S. $971-$ 974.

MATSCHKE, M. J. / BRÖSEL, G. (2003): Zur Bewertung kleiner und mittlerer Unternehmungen mit dem Zustands-Grenzpreismodell unter besonderer Berücksichtigung möglicher Folgen von „Basel II", erscheint in: MEYER, J.-A. (Hrsg.), Unternehmensbewertung von kleinen und mittleren Unternehmen (KMU) - Jahrbuch der KMU-Forschung 2003, München 2003.

MELLEROMTCZ, K. (1952): Der Wert der Unternehmung als Ganzes, Essen 1952.

MENGER, C. (1871): Grundsätze der Volkswirthschaftslehre, Wien 1871.

MOXTER, A. (1983): Grundsätze ordnungsmäßiger Unternehmensbewertung, 2. Aufl., Wiesbaden 1983.

MUNSTERMANN, H. (1966): Wert und Bewertung der Unternehmung, Wiesbaden 1966.

OLBRICH, M. (1999): Unternehmungskultur und Unternehmungswert, Wiesbaden 1999. 
OLBRICH, M. (2000): Zur Bedeutung des Börsenkurses für die Bewertung von Unternehmungen und Untemehmungsanteilen, in: BFuP, 52. Jg. (2000), S. 454-465.

OLBRICH, M. (2002): Zur Unternehmungsnachfolge im elektronischen Geschäft, in: KEUPER, $F$. (Hrsg.), Electronic Business und Mobile Business, Wiesbaden 2002, S. 677-708.

o. $V$. (2002): UMTS-Regeln bleiben unverändert, in: Handelsblatt vom 16. September 2003, S. 18.

o. V. (2003a): Büdelsdorfer Mobilcom will ihr UMTS-Netz notfalls abreißen, in: Handeisblatt vom 24./25. Januar 2003, S. 11.

o. V. (2003b): „Wir haben die Chancen von UMTS eindeutig überschätzt“; in: FAZ vom 28. Januar 2003, S. 14.

PIEPENBROCK, H.-J. / SCHUSTER, F. (Hrsg.) (2001): UMTS-Lizenzvergabe, Baden-Baden 2001.

PFAFF, D. / PFEIFFER, TH. / GATHGE, D. (2002): Unternehmensbewertung und Zustands-Grenzpreismodelle, in: BFuP, 54. Jg. (2002), S. 198-210.

PRIETZE, O. / WALKER, A. (1995): Der Kapitalisierungszinsfuß im Rahmen der Unternehmensbewertung, in: DBW, 55. Jg. (1995), S. 199-211.

REICHERTER, M. (2000): Fusionsentscheidung und Wert der Kreditgenossenschaft, Wiesbaden 2000.

REICHWALD, R. / HERMANN, M. / BIEBERBACH, F. (2000): Auktionen im Internet, in: WISU, 29. Jg. (2000), S. 542-552.

ROLLBERG, R. (1999): Simultane Investitions-, Finanz- und Produktionsprogrammplanung, in: BURCHERT, H. / HERING. TH. (Hrsg.), Betriebliche Finanzwirtschaft, München/Wien 1999, S. $96-110$.

ROLLBERG, R. (2001): Integrierte Unternehmensplanung, Wiesbaden 2001.

SANFLEBER M. (1990): Abfindungsklauseln in Gesellschaftsverträgen, Düsseldorf 1990.

SCHILDBACH, TH. (1995): Der Verkäufer und das Unternehmen ,wie es steht und liegt", in: $\mathrm{ZfbF}, 47$. Jg. (1995), S. 620-632.

SCHMALENBACH, E. (1917/1918): Die Werte von Anlagen und Unternehmungen in der Schätzungstechnik, in: ZfhF, 12. Jg. (1917/1918), S. 1-20.

SERFLING, K. / PAPE, U. (1995): Theoretische Grundlagen und traditionelle Verfahren der Unternehmensbewertung, in: WISU, 24. Jg. (1995), S. 308-819.

SIEBEN, G. (1976): Der Entscheidungswert in der Funktionenlehre der Unternehmensbewertung, in: BFuP, 28. Jg. (1976), S. 491-504

SIEBEN, G. (1988): Untemehmensstrategien und Kaufpreisbestimmung, in: Festschrift 40 Jahre Der Betrieb, Stuttgart 1988. S. 81-91.

SIEBEN, G. (1993): Untemehmensbewertung, in: WITTMANN, W. ET AL. (Hrsg.), Handwörterbuch der Betriebswirtschaft, Teilband 3, 5. Aufl., Stuttgart 1993, Sp. 4315-4331.

SIEBEN, G. / LUTZ. H. (1995): Abfindungsklauseln in Gesellschaftsverträgen, in: BFuP, 47. Jg. (1995), S. 200-213. 
SIEBEN, G. / SCHILDBACH, TH. (1994): Betriebswirtschaftliche Entscheidungstheorie, 4. Aufl., Düsseldorf 1994.

SIEGEL, TH. (1992): Methoden der Unsicherheitsberücksichtigung in der Unternehmensbewertung, in: WiSt, 21. Jg. (1992), S. 21-26.

SIEGMUND. G. (1999): Technik der Netze, 4. Aufl., Heidelberg 1999.

SIELAFF, M. (1977): Die Steuerbemessungsfunktion der Unternehmensbewertung, in: GOETZKE, W. / SIEBEN, G. (Hrsg.), Moderne Unternehmungsbewertung und Grundsätze ihrer ordnungsmäßigen Durchführung, Köln 1977, S. 105-119.

SIEPE, G. (1998): Die Unternehmensbewertung, in: INSTITUT DER WIRTSCHAFTSPRUFER (Hrsg.), Wirtschaftsprüfer-Handbuch 1998, Bd. II, 11. Aufl., Düsseldorf 1998, S. 1-142.

VIEL, J. (1955): Theorie und Praxis der Unternehmungsbewertung, in: Der Wirtschaftstreuhänder, 4. Jg. (1955), S. 57-59.

WEBER, E. (1991): Berücksichtigung von Synergieeffekten bei der Unternehmensbewertung, in: BAETGE, J. (Hrsg.), Akquisition und Unternehmensbewertung, Düsseldorf 1991, S. $97-$ 115. 